PHYSICAL REVIEW D 86, 015002 (2012)

\title{
Phenomenology of anomaly-mediated supersymmetry-breaking scenarios with nonminimal flavor violation
}

\author{
Benjamin Fuks* \\ Institut Pluridisciplinaire Hubert Curien/Département Recherches Subatomiques, \\ Université de Strasbourg/CNRS-IN2P3, 23 Rue du Loess, F-67037 Strasbourg, France \\ Björn Herrmann ${ }^{\dagger}$ \\ LAPTh, Université de Savoie, CNRS, B.P. 110, F-74941 Annecy-le-Vieux, France \\ Michael Klasen \\ Institut für Theoretische Physik, Universität Münster, Wilhelm-Klemm-Straße 9, D-48149 Münster, Germany
}

(Received 19 April 2012; published 5 July 2012)

\begin{abstract}
In minimal anomaly-mediated supersymmetry-breaking models, tachyonic sleptons are avoided by introducing a common scalar mass similar to the one introduced in minimal supergravity. This may lead to nonminimal flavor-violating interactions, e.g., in the squark sector. In this paper, we analyze the viable anomaly-mediated supersymmetry-breaking parameter space in the light of the latest limits on low-energy observables and LHC searches, complete our analytical calculations of flavor-violating supersymmetric particle production at hadron colliders with those related to gluino production, and study the phenomenological consequences of nonminimal flavor violation in anomaly-mediated supersymmetry-breaking scenarios at the LHC. Related cosmological aspects are also briefly discussed.
\end{abstract}

DOI: 10.1103/PhysRevD.86.015002

PACS numbers: 12.60.Jv, 14.80.Da, 14.80.Ly

\section{INTRODUCTION}

Many alternatives to the standard model (SM) of particle physics have been proposed over the last 30 years. Among these, supersymmetry (SUSY), and particularly its minimal version dubbed the minimal supersymmetric standard model (MSSM) [1,2], is one of the most popular SM extensions. It consists in a symmetry linking fields with opposite statistics, matching thus a bosonic (fermionic) superpartner with each fermionic (bosonic) SM degree of freedom. As a consequence, it predicts the stabilization of the gap between the electroweak and the Planck scale, gauge coupling unification at high energies, and a lightest supersymmetric particle (LSP), which is weakly interacting and stable and thus a good dark matter candidate. Since the superpartners of the SM particles have not yet been observed and in order to remain a viable solution to the hierarchy problem, SUSY must be softly broken at low energy, which makes the SUSY particles massive, with a mass lying in the $\mathrm{TeV}$ range. Therefore, SUSY searches at present hadron colliders, such as the Tevatron at Fermilab or the LHC at CERN, are important topics of the current experimental high-energy physics program.

Within the standard model, flavor violation in the quark sector arises only through the rotation of the up- and downtype quark interaction eigenstates into the basis of physical mass eigenstates. Four biunitary matrices are required to diagonalize the quark Yukawa matrices, which renders the

\footnotetext{
*benjamin.fuks@iphc.cnrs.fr

†herrmann@in2p3.fr

michael.klasen@uni-muenster.de
}

charged-current interactions proportional to the unitary Cabibbo-Kobayashi-Maskawa (CKM) quark-mixing matrix $V_{\mathrm{CKM}}$. In the super-CKM basis [3], the squark interaction eigenstates undergo the same rotations as their quark counterparts, so that their charged-current interactions are also proportional to the CKM matrix. However, in nonminimal flavor-violating (NMFV) supersymmetric theories, the quark and squark fields can be misaligned due to additional sources of flavor violation which are related to the breaking of supersymmetry. As a consequence, this leads to flavorviolating (nondiagonal) entries in the squark mass matrices.

In recent works, we have analyzed the cases where such soft terms appear in (nonminimal) supergravity and gaugemediated SUSY-breaking scenarios [4,5]. In the first case, supersymmetry is broken in a hidden sector and transmitted to the visible sector of squarks, sleptons, gauginos and gluinos through gravitational interactions. Soft masses for sfermions are induced by direct Kähler interactions, which can in general be flavor nondiagonal [1,6,7]. In the second case, the breaking of supersymmetry is mediated to the visible sector via gauge interactions with messenger fields in a flavor-conserving fashion [8-11]. However, it has recently been shown that nonminimal versions of the gaugemediated SUSY-breaking mechanism can yield important flavor violation in the squark and slepton sectors [11-13].

When SUSY is broken in a hidden sector, the soft masses also receive contributions from quantum effects due to the superconformal anomaly [14-17]. In this work, we therefore consider this so-called anomaly-mediated SUSY-breaking (AMSB) scenario, where those anomaly-mediated effects are large compared to all other sources of SUSY breaking, 
which are subdominant. We extend our previous work on flavor violation $[4,5]$ by investigating possible nonminimal flavor violation within the AMSB context.

This paper is organized as follows: In Sec. II, we define anomaly-mediated supersymmetry-breaking scenarios and show how nonminimal flavor violation can appear. In Sec. III, we impose current experimental constraints on the flavor-violating AMSB scenario and perform scans of the parameter space. In addition, experimentally allowed benchmark points are defined. Cross sections for the production of at least one gluino are analytically and numerically computed in Sec. IV. We dedicate Sec. V to an analysis of the possible cosmological constraints related to the presence of cold dark matter in our Universe. Our conclusions are presented in Sec. VI.

\section{ANOMALY MEDIATION AND FLAVOR VIOLATION IN THE SQUARK SECTOR}

In AMSB scenarios, the soft terms are related to the anomalous dimensions of the different fields and have the feature to be renormalization-group invariant $[18,19]$. As a

consequence, they are fully determined by the known low-energy gauge and Yukawa couplings and an overall mass scale $m_{\text {aux }}$, the vacuum expectation value of the scalar auxiliary field of the gravitation supermultiplet. This scale is expected to be of the order of the gravitino mass $m_{3 / 2}$, and we assume in the following, to simplify, $m_{3 / 2}=m_{\text {aux }}$. Consequently, the model is highly predictive, with fixed mass ratios and distinctive signatures [16,20-23]. Among all the predictions, one finds, however, tachyonic sleptons. This problem must be cured in order to have a phenomenologically viable model. Several solutions have been proposed [14,17,24-33], and we adopt here the phenomenological approach of assuming non-negligible contributions to the scalar soft masses, induced, e.g., by supergravity, which makes their square positive at the weak scale. However, in this case, solving the tachyonic sfermion mass problem can also introduce nonminimal flavor violation in the theory, through possible nondiagonal flavor-violating soft mass terms.

The squark mass matrices are written, in the super-CKM basis, as

$$
M_{\tilde{q}}^{2}=\left(\begin{array}{ccccccc}
M_{L_{q_{1}}}^{2} & \Delta_{L L}^{q_{1} q_{2}} & \Delta_{L L}^{q_{1} q_{3}} & \mid & m_{q_{1}} X_{q_{1}} & \Delta_{L R}^{q_{1} q_{2}} & \Delta_{L R}^{q_{1} q_{3}} \\
\Delta_{L L}^{q_{1} q_{2} *} & M_{L_{q_{2}}}^{2} & \Delta_{L L}^{q_{2} q_{3}} & \mid & \Delta_{R L}^{q_{1} q_{2} *} & m_{q_{2}} X_{q_{2}} & \Delta_{L R}^{q_{2} q_{3}} \\
\Delta_{L L}^{q_{1} q_{3^{*}}} & \Delta_{L L}^{q_{2} q_{3} *} & M_{L_{q_{3}}}^{2} & \mid & \Delta_{R L}^{q_{1} q_{3}^{*}} & \Delta_{R L}^{q_{2} q_{3} *} & m_{q_{3}} X_{q_{3}} \\
\overline{m_{q_{1}} X_{q_{1}}^{*}} & \overline{\Delta_{R L}^{q_{1} q_{2}}} & \overline{\Delta_{R L}^{q_{1} q_{3}}} & \mid & \overline{M_{R_{q_{1}}}^{2}} & \overline{\Delta_{R R}^{q_{1} q_{2}}} & \overline{\Delta_{R R}^{q_{1} q_{3}}} \\
\Delta_{L R}^{q_{1} q_{2} *} & m_{q_{2}} X_{q_{2}}^{*} & \Delta_{R L}^{q_{2} q_{3}} & \mid & \Delta_{R R}^{q_{1} q_{2} *} & M_{R_{q_{2}}}^{2} & \Delta_{R R}^{q_{2} q_{3}} \\
\Delta_{L R}^{q_{1} q_{3^{*}}} & \Delta_{L R}^{q_{2} q_{3} *} & m_{q_{3}} X_{q_{3}}^{*} & \mid & \Delta_{R R}^{q_{1} q_{3} *} & \Delta_{R R}^{q_{2} q_{3} *} & M_{R_{q_{3}}}^{2}
\end{array}\right),
$$

where the flavor-diagonal elements are given by

$$
\begin{aligned}
M_{L_{q_{i}}}^{2} & =M_{\tilde{Q}_{i}}^{2}+m_{q_{i}}^{2}+\cos 2 \beta m_{Z}^{2}\left(T_{q}^{3}-e_{q} s_{W}^{2}\right), \\
M_{R_{q_{i}}}^{2} & =M_{\tilde{U}_{i}}^{2}+m_{q_{i}}^{2}+\cos 2 \beta m_{Z}^{2} e_{q} s_{W}^{2} \quad \text { for up-type squarks, } \\
M_{R_{q_{i}}}^{2} & =M_{\tilde{D}_{i}}^{2}+m_{q_{i}}^{2}+\cos 2 \beta m_{Z}^{2} e_{q} s_{W}^{2} \quad \text { for down-type squarks, } \\
X_{q_{i}} & =A_{q_{i}}^{*}-\mu \begin{cases}\cot \beta & \text { for up-type squarks, } \\
\tan \beta & \text { for down-type squarks. }\end{cases}
\end{aligned}
$$

The weak isospin quantum numbers are $T_{q}^{3}= \pm 1 / 2$ for left-handed up-type and down-type (s)quarks, their fractional electromagnetic charge is denoted by $e_{q}$, and $m_{q_{i}}$ is the mass of the quark $q_{i}, i$ being the flavor index, i.e., $d_{1}=d, d_{2}=s, d_{3}=b, u_{1}=u, u_{2}=c$, and $u_{3}=t$. In addition, $m_{Z}$ is the $Z$-boson mass, and $s_{W}$ is the sine of the electroweak mixing angle. The soft supersymmetrybreaking mass terms are $M_{\tilde{Q}_{i}}$ and $M_{\left\{\tilde{U}_{i}, \tilde{D}_{i}\right\}}$ for the lefthanded and right-handed squarks, while the quantities $A_{q_{i}}$ are the trilinear couplings between the Higgs bosons and the scalar SUSY particles. In the Higgs sector, $\mu$ denotes the off-diagonal superpotential Higgs mass parameter, and $\tan \beta=v_{u} / v_{d}$ is the ratio of vacuum expectation values of the two Higgs doublets. In our phenomenological approach, the off-diagonal parameters $\Delta_{a b}^{q q^{\prime}}$ are arbitrary and can be normalized to the diagonal entries according to [34]

$$
\Delta_{a b}^{q_{i} q_{j}}=\lambda_{a b}^{q_{i} q_{j}} M_{q_{i}} M_{q_{j}} .
$$

Additional sources of quark flavor violation are then parametrized through the 21 dimensionless (possibly complex) new variables $\lambda_{a b}^{q_{q} q_{j}}$, since due to $S U(2)$ gauge invariance, the $\Delta_{L L}^{q q^{\prime}}$ elements of the up- and down-type squark squared mass matrices are related to each other, 


$$
M_{\tilde{u}, L L}^{2}=V_{\mathrm{CKM}} M_{\tilde{d}, L L}^{2} V_{\mathrm{CKM}}^{\dagger},
$$

as are the associated $\lambda$ parameters. This equation shows that both squark mass matrices cannot be simultaneously diagonal (without neglecting the CKM matrix). The diagonalization of the mass matrices $M_{\tilde{u}}^{2}$ and $M_{\tilde{d}}^{2}$ requires the introduction of two $6 \times 6$ matrices $R^{u}$ and $R^{d}$,

$$
\begin{aligned}
& \operatorname{diag}\left(m_{\tilde{u}_{1}}^{2}, \ldots, m_{\tilde{u}_{6}}^{2}\right)=R^{u} M_{\tilde{u}}^{2} R^{u \dagger} \quad \text { and } \\
& \operatorname{diag}\left(m_{\tilde{d}_{1}}^{2}, \ldots, m_{\tilde{d}_{6}}^{2}\right)=R^{d} M_{\tilde{d}}^{2} R^{d \dagger},
\end{aligned}
$$

where by convention the masses are ordered increasingly, $m_{\tilde{q}_{1}}<\ldots<m_{\tilde{q}_{6}}$. These mixing matrices relate the physical mass eigenbasis to the interaction eigenbasis through

$\left(\tilde{u}_{1}, \tilde{u}_{2}, \tilde{u}_{3}, \tilde{u}_{4}, \tilde{u}_{5}, \tilde{u}_{6}\right)^{t}=R^{u}\left(\tilde{u}_{L}, \tilde{c}_{L}, \tilde{t}_{L}, \tilde{u}_{R}, \tilde{c}_{R}, \tilde{t}_{R}\right)^{t}$ and

$\left(\tilde{d}_{1}, \tilde{d}_{2}, \tilde{d}_{3}, \tilde{d}_{4}, \tilde{d}_{5}, \tilde{d}_{6}\right)^{t}=R^{d}\left(\tilde{d}_{L}, \tilde{s}_{L}, \tilde{b}_{L}, \tilde{d}_{R}, \tilde{s}_{R}, \tilde{b}_{R}\right)^{t}$.

Recently, it has been shown that (minimal) AMSB scenarios with an additional $U(1)$ symmetry satisfy the requirements of the minimal flavor-violation principles [35]. In this case, the $\lambda$ parameters are not free and directly dictated from the flavor structure of the CKM matrix. In our approach, we are going beyond this scheme, keeping the flavor-violation parameters independent.

\section{EXPERIMENTAL CONSTRAINTS ON AMSB MODELS}

\section{A. Constraints}

In this section, we discuss the most relevant experimental measurements that can be used to constrain the parameter space of the MSSM. Apart from direct searches for superpartners at collider experiments, and particularly the recent results of the ATLAS and CMS experiments at the LHC [36,37], numerous low-energy and electroweak precision measurements $[38,39]$ constrain masses and mixings of the superpartners. They can often impose stronger limits on the flavor-violating entries introduced in Sec. II.

Extensive studies of the kaon sector, $B$ - and $D$-meson oscillations, rare decays, and electric dipole moments suggest that only flavor mixing involving the second and third generations of squarks can be substantial, and this only in the left-left and right-right chiral sectors, which mix the superpartners of the left-handed and right-handed quarks [34,40-42]. For this reason, we restrict ourselves to the simpler scenario where only the flavor-mixing parameters related to the second and third generations and nonmixing squark chiralities can be nonvanishing,

$$
\lambda_{\mathrm{L}} \equiv \lambda_{L L}^{c t}, \quad \lambda_{u} \equiv \lambda_{R R}^{c t} \quad \text { and } \quad \lambda_{d} \equiv \lambda_{R R}^{s b} .
$$

The parameter related to the mixing among second- and third-generation down-type squarks $\lambda_{L L}^{s b}$ is not a free parameter of our simplified model, since it is connected to $\lambda_{L}$ through Eq. (2.4).
The measurement of the rare $b \rightarrow s \gamma$ decay represents one of the most stringent constraints on these squark mixings. The inclusive branching ratio (BR) is determined to be

$$
\mathrm{BR}(b \rightarrow s \gamma)=\left(3.55 \pm 0.26_{\text {exp }} \pm 0.23_{\text {theo }}\right) \times 10^{-4}
$$

from BABAR, BELLE, and CLEO data [39]. For the theoretical error estimate, we refer to the discussion in Refs. $[43,44]$. Squark diagrams contribute already at the one-loop level, as do the SM particles. As a consequence, this measurement can lead to strong constraints on the squark masses and couplings, especially on the $\lambda_{\mathrm{L}}$ parameter of the left-left chiral sector, since the lightest neutralinos and charginos appearing in the loops are mostly winos coupling to the left-handed components of the squark mass eigenstates. The same arguments apply to the $b \rightarrow s \mu^{+} \mu^{-}$branching fraction, experimentally measured as [39]

$$
\mathrm{BR}\left(b \rightarrow s \mu^{+} \mu^{-}\right)=\left(2.23 \pm 0.98_{\exp } \pm 0.11_{\text {theo }}\right) \times 10^{-6} \text {, }
$$

the associated theoretical uncertainties being computed in Ref. [45], or to the $B_{s}^{0}$-meson branching fractions to a muon pair, recently bounded from above by the CMS and LHCb experiments [46],

$$
\mathrm{BR}\left(B_{s}^{0} \rightarrow \mu^{+} \mu^{-}\right)<1.1 \times 10^{-8},
$$

at $95 \%$ confidence level, which is, however, still about 4 or 5 times larger than the SM expectation.

Also, the $B_{s}^{0}-\bar{B}_{s}^{0}$ oscillations, which have been recently observed, directly probe the mixing between squarks of the second and third generations. Since NMFV contributions arise at the same loop level as the SM ones, this observable can again be sensitive to nonvanishing $\lambda$ parameters. Hence, the measured mass difference [39]

$$
\Delta M_{B_{s}^{0}}=\left(17.78 \pm 0.12_{\exp } \pm 3.3_{\text {theo }}\right) \mathrm{ps}^{-1},
$$

where the theoretical uncertainty of $3.3 \mathrm{ps}^{-1}$ at the 95\% confidence level has been derived in Ref. [47], allows to constrain the magnitude of the above-mentioned flavorviolating parameters.

Another important consequence of NMFV mixing in the squark sector is a large splitting between squark mass eigenvalues. This influences the $Z$ - and $W$-boson selfenergies at zero momentum, contributing hence to the electroweak $\rho$ parameter

$$
\begin{aligned}
\Delta \rho & =\frac{\Sigma_{Z}(0)}{m_{Z}^{2}}-\frac{\Sigma_{W}(0)}{m_{W}^{2}}=\alpha\left(m_{Z}\right) T \\
& =(2.36 \pm 8.65) \times 10^{-4},
\end{aligned}
$$

the experimental value arising from combined fits of the $Z$-boson mass, width, and pole asymmetry as well as of the masses of the $W$ boson and the top quark [38].

Furthermore, recent measurements of the anomalous magnetic moment of the muon $(g-2)_{\mu}$ indicate a discrepancy of about $3 \sigma$ between the data and the standard model predictions [38], 


$$
\begin{aligned}
a_{\mu}^{\exp }-a_{\mu}^{\mathrm{SM}} & =(25.5 \pm 7.98) \times 10^{-10} \quad \text { with } \\
a_{\mu} & \equiv(g-2)_{\mu} / 2 .
\end{aligned}
$$

This gap could be explained by new physics. In the case of supersymmetric scenarios, the leading contributions, depending on the smuon, sneutrino, chargino and neutralino masses, have been found to be proportional to the sign of the $\mu$ parameter [48]. Since negative values would then increase the discrepancy, we limit ourselves to positive values of $\mu$. Moreover, since squarks contribute only at the two-loop level, the dependence on flavor violation in the squark sector is expected to be considerably reduced. Finally, from direct searches of the Higgs boson, we ask for the mass of the lightest Higgs boson to fulfil

$$
111 \mathrm{GeV} \lesssim m_{h} \lesssim 130 \mathrm{GeV} \text {. }
$$

The lower bound is based on the exclusion limit of $m_{h}<$ 114.4 GeV from CERN LEP [38], after accounting for a theoretical uncertainty of about $3 \mathrm{GeV}$ [49], while the upper bound corresponds to the nonobservation of a Higgs boson by the ATLAS and CMS experiments [50,51]. For a restricted set of universal scalar mass $m_{0}$, introduced to solve the tachyonic slepton problem, smaller than a few $\mathrm{TeV}$, the upper bound is almost always satisfied. Considering quantum corrections, the Higgs mass $m_{h}$ depends on the squark masses, and thus on the flavorviolating entries in the associated mass matrices.

\section{B. Analysis of the parameter space related to AMSB scenarios with nonminimal flavor violation}

In order to study the phenomenology of AMSB models including nonminimal flavor violation, we have scanned over the parameter space of the model, generalizing the squark mass matrices by including the three flavorviolation parameters presented in Eq. (3.1). Our procedure starts at a high-energy scale, where we define our input parameters as the gravitino mass $m_{3 / 2}$, the universal scalar mass $m_{0}$ introduced to solve the tachyonic slepton problem, the ratio of the two neutral Higgs field vacuum expectation values $\tan \beta=v_{u} / v_{d}$, and the sign of the Higgs mixing parameter $\operatorname{sgn}(\mu)$. The soft supersymmetry-breaking terms at the electroweak scale are then obtained through renormalization group running using the SPHENO package version 3.0 [49], which solves the renormalization-group equations numerically to two-loop order and extracts the particle spectrum and mixings at the electroweak scale at the one-loop level for the matter and gauge sectors and at the two-loop level for the Higgs sector. It also computes the electroweak precision and low-energy observables presented in Sec. III A.

For the numerical values of the standard model parameters, we fix the top-quark pole mass to $m_{\text {top }}^{\text {pole }}=173.2 \mathrm{GeV}$ [52], the bottom-quark mass to $m_{b}\left(m_{b}\right)=4.2 \mathrm{GeV}$ and the $Z$-boson mass to $m_{Z}=91.1876 \mathrm{GeV}$. The Fermi constant has been taken as $G_{F}=1.16637 \times 10^{-5} \mathrm{GeV}^{-2}$, and the strong and electromagnetic coupling constants at the $Z$ pole as $\alpha_{s}\left(m_{Z}\right)=0.1176$ and $\alpha\left(m_{Z}\right)^{-1}=127.934$. At this stage, the only source of flavor violation lies within the CKM matrix, which is calculated using the Wolfenstein parametrization. The corresponding four free parameters are set to $\lambda^{(\mathrm{CKM})}=0.2272, A^{(\mathrm{CKM})}=0.818, \bar{\rho}^{(\mathrm{CKM})}=0.221$, and $\bar{\eta}^{(\mathrm{CKM})}=0.34$ [38].

In Fig. 1, we present typical scans of the minimal AMSB parameter space, i.e., when flavor violation is induced by
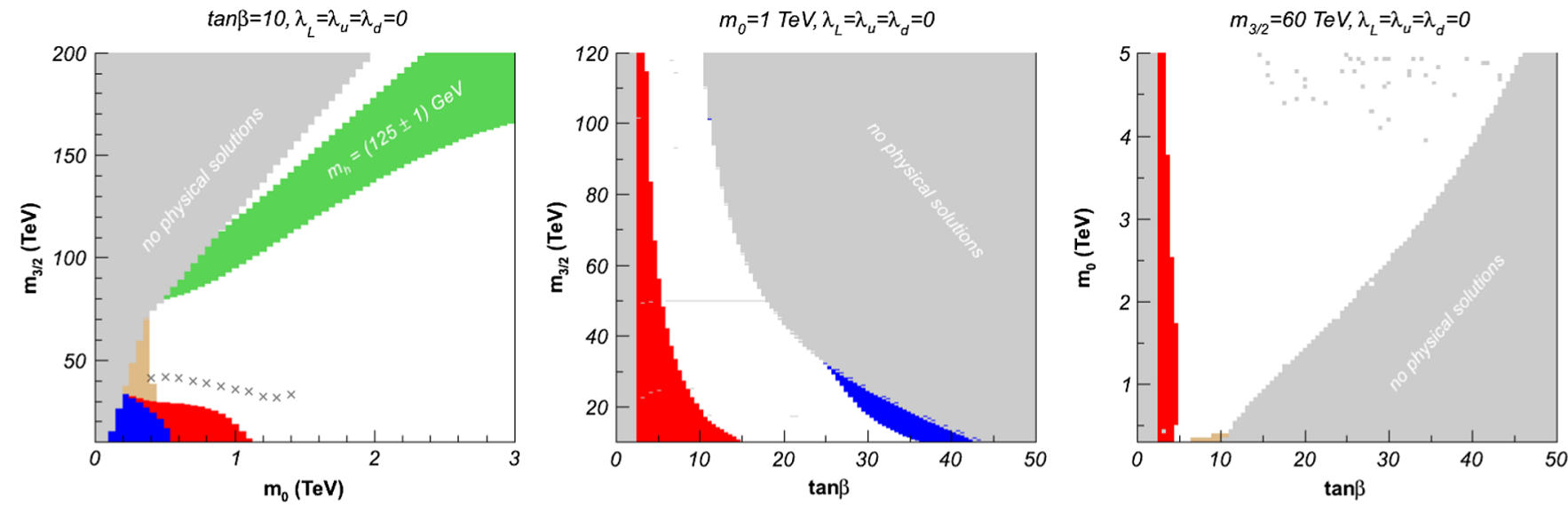

FIG. 1 (color online). Scans of the minimal AMSB parameter space, where all flavor-violating parameters, apart from the CKM matrix, vanish, in the $\left(m_{0}, m_{3 / 2}\right)$ plane at fixed $\tan \beta=10$ (left panel), in the ( $\left.\tan \beta, m_{3 / 2}\right)$ plane at fixed $m_{0}=1 \mathrm{TeV}$ (central panel) and in the $\left(\tan \beta, m_{0}\right)$ plane at fixed $m_{3 / 2}=60 \mathrm{TeV}$ (right panel). We show regions where there is no physical solution to the renormalization-group equations (grey), or which are excluded by the constraints related to the $b \rightarrow s \gamma$ branching ratio (blue) and the Higgs mass (red). The regions, where the agreement between theory and experiment for the anomalous magnetic moment of the muon is restored, are presented in beige. On the left panel, we also indicate the region where the mass of the lightest Higgs boson is close to $125 \mathrm{GeV}$ (green) and the exclusion limit obtained (crosses) from the reinterpretation of the LHC results on supersymmetric particle searches in the context of AMSB scenarios (see Ref. [53]). 
the CKM matrix alone and all $\lambda$ parameters vanish. We show the examples of the $\left(m_{0}, m_{3 / 2}\right)$ plane at fixed $\tan \beta=$ 10 (left panel), the $\left(\tan \beta, m_{3 / 2}\right)$ plane at fixed $m_{0}=1 \mathrm{TeV}$ (central panel) as well as the $\left(\tan \beta, m_{0}\right)$ plane at fixed $m_{3 / 2}=60 \mathrm{TeV}$ (right panel). All experimental limits described in Sec. III A are imposed at the $2 \sigma$ level. We observe that the low-mass regions with a relatively small $m_{0}$, attractive from a collider point of view, are strongly disfavored by both the measurements of the $b \rightarrow s \gamma$ branching ratio and the too low predicted mass for the lightest Higgs boson. The latter also excludes small values of $\tan \beta$ for a large range of $m_{0}$ and $m_{3 / 2}$ masses. In addition, a significant part of the regions where the predicted value for the $b \rightarrow s \gamma$ branching ratio lies outside the $2 \sigma$ range deduced from Eq. (3.2) is also excluded by the constraints associated to the $b \rightarrow s \mu^{+} \mu^{-}$branching ratio. Therefore, these regions have not been shown on Fig. 1, for clarity. Predictions for the other considered $B$-physics observables, $\Delta M_{B_{s}^{0}}$ and the branching ratio $B_{s}^{0} \rightarrow \mu^{+} \mu^{-}$, are mostly compatible with data and hence absent from the figure, as is the computed value for the $\Delta \rho$ parameter. This quantity only restricts the parameter space at very heavy masses, which lie outside the mass range presented in the figures.

On the left panel of Fig. 1, we also show the exclusion limits on the parameter space of AMSB scenarios obtained from a reinterpretation of the results of the direct searches for superparticles at the LHC in the AMSB context [53], as well as the region where the mass of the lightest Higgs boson is close to $125 \mathrm{GeV}$, a value favored by the recent observations of the ATLAS and CMS experiments [50,51]. However, in the absence of a confirmed signal for a Higgs boson in that mass range, we do not consider this last limit for our analysis in the sequel.

The size of the regions, where the gap between the data and the predictions for the anomalous magnetic moment of the muon $a_{\mu}$ is closed, is relatively small. Moreover, a significant fraction of it is excluded by the $b \rightarrow s \gamma$ constraint, as it has also been found in Refs. [35,54]. Hence, a possible explanation of the discrepancy between theory and data for this observable by contributions related to AMSB scenarios is rather difficult. However, the dominant supersymmetric contributions to $a_{\mu}$ highly depend on the slepton masses, entering into the theoretical calculation already at the one-loop level. Since in the context of AMSB scenarios, any prediction associated to the slepton sector is tightly linked to the employed solution to solve the tachyonic slepton problem, we choose to relax the constraint associated to the anomalous magnetic moment of the muon. This is also motivated by the fact that nonminimal flavor violation in the squark sector, i.e., the scope of this paper, contributes subdominantly, at the two-loop level, to $a_{\mu}$, so that it becomes almost independent of the $\lambda$ parameters as it was already noticed in the discussions of Refs. [4,5].
We now turn to nonminimal flavor violation in AMSB scenarios, where nonvanishing $\lambda$ parameters can arise, e.g., from nontrivial Kähler interactions as in supergravity. In our phenomenological approach, we introduce nonminimal flavor violation at the weak scale, generalizing the squark mass matrices by introducing the three parameters defined in Eq. (3.1). We use again the SPHENO program to diagonalize the squark sector and compute the flavor and weak observables presented in Sec. III A. In Figs. 2 and 3, we depict the impact of the considered NMFV parameters on these observables, showing the associated constraints on the nonminimal AMSB parameter space. We impose the above-mentioned limit for the Higgs-boson mass, i.e., $m_{h} \geq 111 \mathrm{GeV}$ (see the discussion in Sec. III A), as well as all the other constraints at the $2 \sigma$ confidence level. We present different typical AMSB planes for different values of the flavor-violating $\lambda$ parameters, keeping only the most constraining observables for clarity and neglecting the anomalous magnetic moment of the muon for the reasons mentioned above.

Typical scans in the $\left(m_{0}, m_{3 / 2}\right)$ planes of the AMSB parameter space are shown in Fig. 2. We use a fixed value of $\tan \beta=10$ and choose a positive sign for the $\mu$ parameter. Comparing with the corresponding minimal AMSB results (left panel of Fig. 1), we find that the most sensitive observable is the $b \rightarrow s \gamma$ branching ratio, which directly probes squark mixing in the left-left chiral sector, i.e., the $\lambda_{L}$ parameter. This strong dependence is due to squark and neutralino-chargino loops, involving $S U(2)_{L}$ interactions between squarks, quarks and neutralinos or charginos, proportional to the squark mixing matrices $R^{u}$ and $R^{d}$ [see Eq. (4.5) below]. This also explains the less pronounced sensitivity to NMFV mixing in the right-right chiral sectors $\left(\lambda_{u}\right.$ and $\left.\lambda_{d}\right)$, where the results do not show any strong dependence on the $\lambda$ parameters. At intermediate values of $\lambda_{\mathrm{L}} \sim 0.1$, the interplay between squark masses and mixings is such that almost all the parameter space accessible by renormalization group running remains allowed by the $b \rightarrow s \gamma$ branching ratio compared to the minimal results of Fig. 1. Similar conclusions hold for the $b \rightarrow s \mu \mu$ branching ratio, not presented on the figures for clarity. The other considered observables, e.g., the constraint on the lightest Higgs-boson mass, are barely sensitive to nonminimal flavor violation, as the dependence on the squark sector is subdominant.

In Fig. 3, we investigate the dependence of the predictions for weak and flavor observables on $\tan \beta$. We first fix the universal scalar mass $m_{0}$ to $1 \mathrm{TeV}$ and scan over the $\left(\tan \beta, m_{3 / 2}\right)$ plane. Secondly, we fix the gravitino mass $m_{3 / 2}$ to $60 \mathrm{TeV}$ and scan over the $\left(\tan \beta, m_{0}\right)$ plane. In both cases, the sign of the $\mu$ parameter is again chosen to be positive. As for the analysis in Fig. 2, the most sensitive observables to nonminimal flavor violation are the $b \rightarrow s \gamma$ and $b \rightarrow s \mu \mu$ branching ratios. Since the constraints related to the second decay are weaker and 

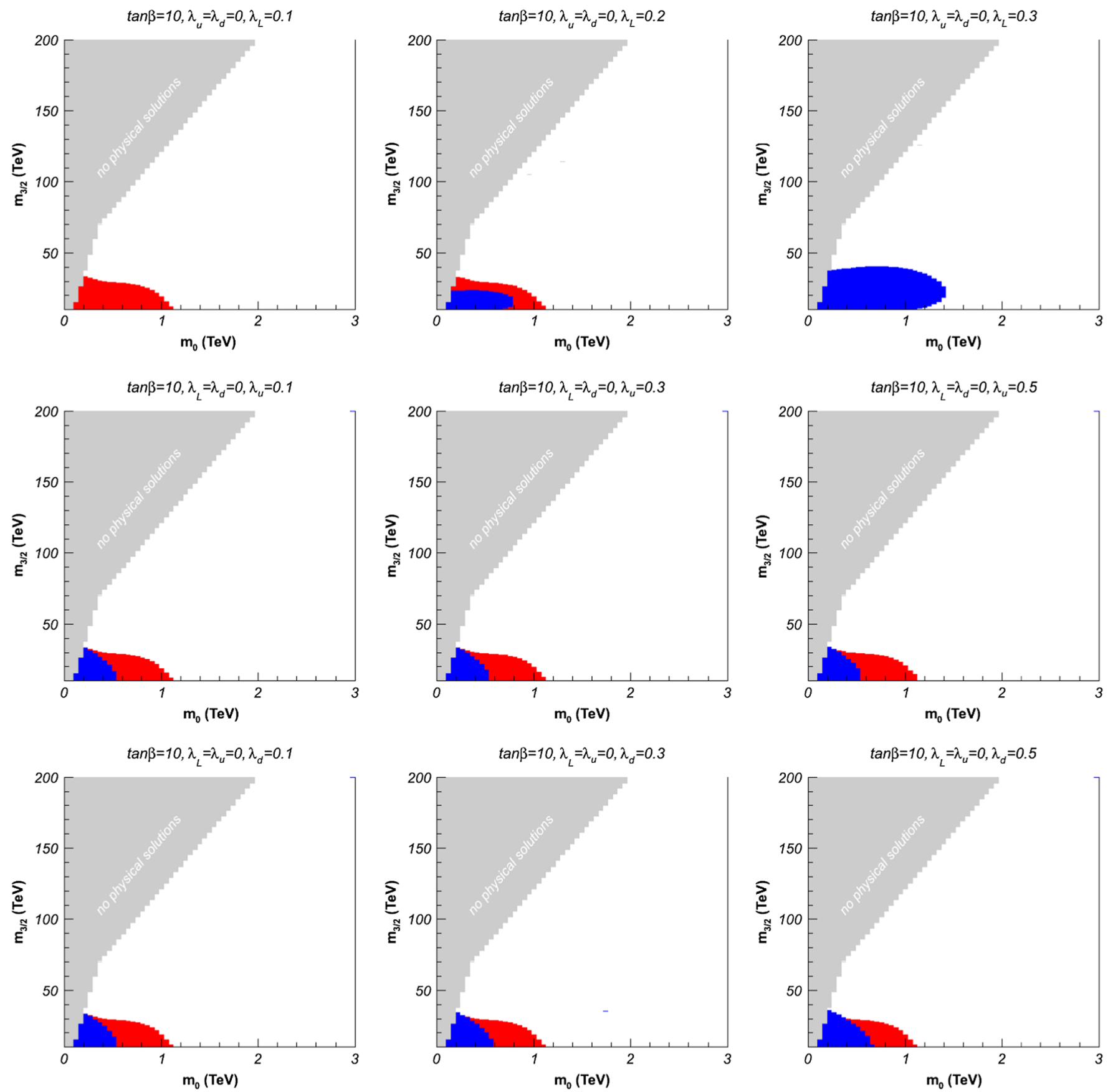

FIG. 2 (color online). Typical scans of NMFV AMSB scenarios in the $\left(m_{0}, m_{3 / 2}\right)$ plane with a fixed value of tan $\beta=10$. The sign of the $\mu$ parameter is chosen positive and we present the results for different values of the NMFV parameters $\lambda_{\mathrm{L}}$ (upper panels), $\lambda_{u}$ (central panels) and $\lambda_{d}$ (lower panels). We show regions excluded due to the absence of physical solutions to the renormalization-group equations (grey), by the constraints associated to the $b \rightarrow s \gamma$ branching ratio (blue) and the Higgs mass (red).

overlapping with those of the first, they are again omitted from the figures. The regions of the parameter space excluded by the constraints associated to the $b \rightarrow s \gamma$ branching ratio increase with the values of the flavor-violating parameter $\lambda_{\mathrm{L}}$. For small values, squark mixings and mass effects compensate, which leaves again almost all the parameter space open. In fact, the exclusion contours related to the $b \rightarrow s \gamma$ branching ratio even vanish, when $\lambda_{\mathrm{L}}$ increases slightly. Such contours reappear only at much larger $\lambda_{\mathrm{L}}$ values. As a consequence, for $\tan \beta \geqslant 20$, possible flavor-violating entries in the squark mass matrices are reduced to be rather modest, i.e., $\lambda_{\mathrm{L}} \leqslant 0.15$. On the other hand, very low values of $\tan \beta$ are excluded by the bounds on the lightest Higgs-boson mass for all values of 

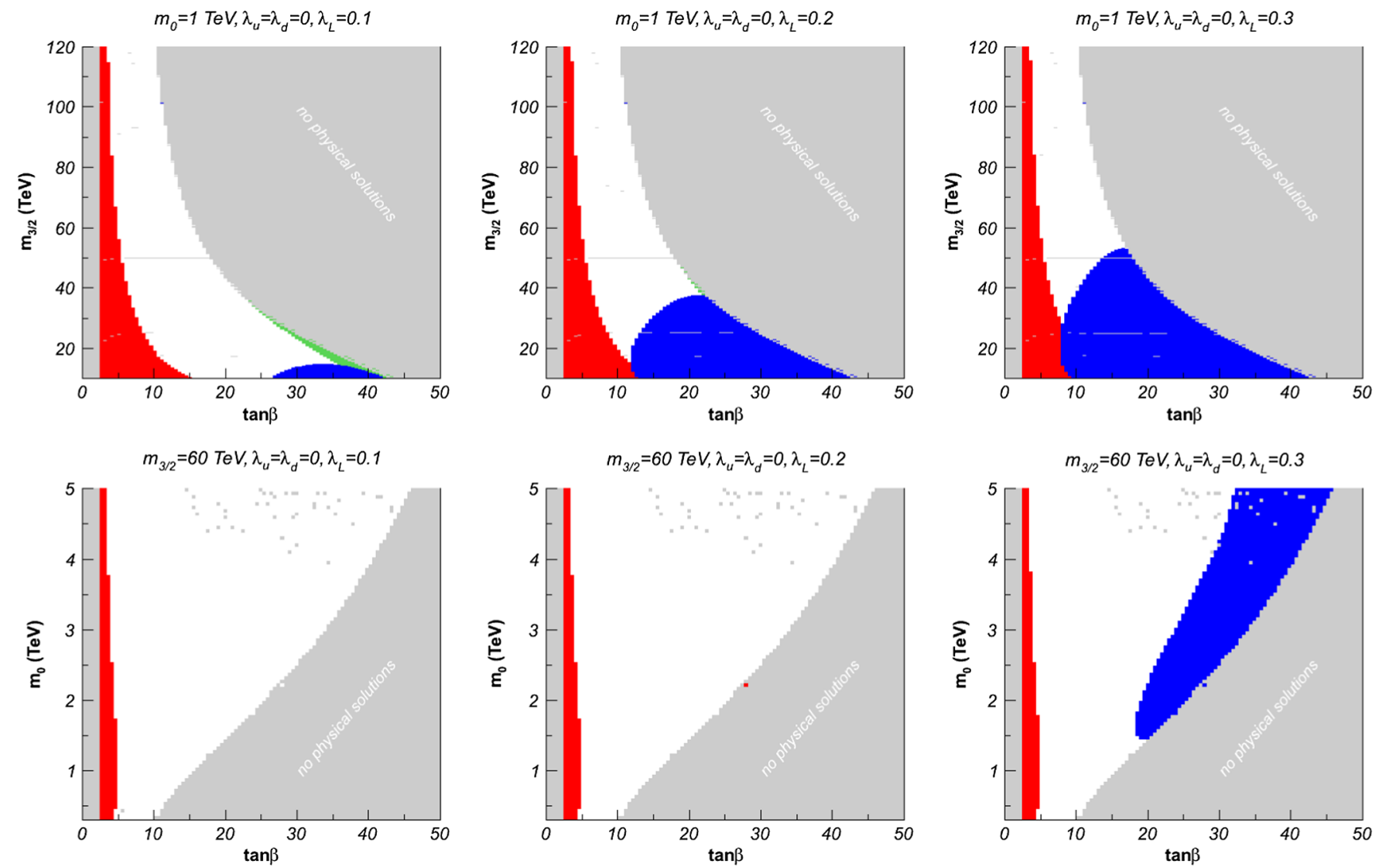

FIG. 3 (color online). Scans of NMFV AMSB scenarios in the $\left(\tan \beta, m_{3 / 2}\right)$ plane with a fixed value of $m_{0}=1$ TeV (upper panels) and in the $\left(\tan \beta, m_{0}\right)$ plane with a fixed value of $m_{3 / 2}=60 \mathrm{TeV}$ (lower panels). The sign of the $\mu$ parameter is chosen positive and we present the results for different values of the NMFV parameter $\lambda_{L}$. We show regions excluded due to the absence of physical solutions to the renormalization-group equations (grey), by the constraints associated to the $b \rightarrow s \gamma$ branching ratio (blue), the $B_{s}^{0}$-meson branching to a muon pair $\operatorname{BR}\left(B_{s}^{0} \rightarrow \mu^{+} \mu^{-}\right)$(green) and the lightest Higgs-boson mass (red).

$\lambda_{\mathrm{L}}$. It is also interesting to remark that the predictions for the $\operatorname{BR}\left(B_{s}^{0} \rightarrow \mu^{+} \mu^{-}\right)$observable lie above the current experimental bounds of Eq. (3.4) in the rather large $\tan \beta$ region, for moderate values of the mixing parameter of the left-left chiral sector $\left(\lambda_{\mathrm{L}} \in[0.1-0.2]\right)$ and for relatively light gravitino and universal scalar masses.

Let us finally note that the results for nonminimal flavor violation in the right-right chiral sector are unchanged with respect to the minimal case shown in Fig. 1, and we subsequently do not present the corresponding figures.

\section{Benchmark scenarios for nonminimally flavor-violating AMSB scenarios}

Inspecting the various AMSB planes presented in Sec. III B, we select three benchmark scenarios allowed by the present low-energy and electroweak precision constraints, which permit a sizeable mixing between second- and third-generation squarks and which are colliderfriendly in the sense that one or several superpartners could be produced with a large rate at the LHC. This means that at least some of the superpartners should not be too heavy.
The chosen benchmark points are presented in Table I, together with the SPS9 benchmark point [55] which is shown as a reference. As a generic feature for all the four points, the gravitino mass is taken as $m_{3 / 2}=60 \mathrm{TeV}$ and the sign of the off-diagonal Higgs mixing parameter is positive, for the reasons discussed in Sec. III A. Because of the renormalization-group running invariance of the gaugino mass parameters $M_{1}, M_{2}$ and $M_{3}$, the dependence of the masses of the gluino, the two lightest neutralinos and the lightest chargino, all mainly gauginolike, on $m_{0}$ and $\tan \beta$ is reduced and subdominant. Therefore, they are roughly identical for all scenarios, with $m_{\tilde{g}} \sim$ $1300-1400 \mathrm{GeV}, \quad m_{\tilde{\chi}_{1}^{0}} \sim m_{\tilde{\chi}_{1}^{ \pm}} \sim 175 \mathrm{GeV}, \quad$ and $m_{\tilde{\chi}_{2}^{0}} \sim$ $550 \mathrm{GeV}$. In contrast, the scalar spectrum is quite different.

The point SPS9 presents a low value of $\tan \beta=10$ as well as a relatively low sfermion mass parameter $m_{0}=$ $450 \mathrm{GeV}$. Therefore, the masses of the color-neutral scalar partners of the SM fermions remain rather moderate, while the squark masses are comparable to the gluino mass. The lightest Higgs-boson mass, $m_{h^{0}}=118.4 \mathrm{GeV}$, lies well within the limit of Eq. (3.8). Our first benchmark point I 
TABLE I. Three AMSB benchmark scenarios allowing for sizeable flavor-violating entries in the squark mass matrices with respect to the second- and third-generation mixing in the left-left and/or the right-right chiral squark sectors. The SPS9 scenario is also presented for comparison. We indicate the SUSY-breaking parameters at the high scale and the resulting masses at low energy (after renormalization group running) for the gluino, the lightest squarks and sleptons, the two lightest neutralinos, the lightest chargino, and the lightest Higgs boson. The values are presented assuming constrained minimal flavor violation, i.e., when $\lambda_{L}=$ $\lambda_{u}=\lambda_{d}=0$.

\begin{tabular}{|c|c|c|c|c|c|c|c|c|c|c|c|c|c|}
\hline & \multirow[b]{2}{*}{$m_{3 / 2}[\mathrm{TeV}]$} & \multirow[b]{2}{*}{$m_{0}[\mathrm{TeV}]$} & \multirow[b]{2}{*}{$\tan \beta$} & \multirow[b]{2}{*}{$\operatorname{sgn}(\mu)$} & \multicolumn{9}{|c|}{$[\mathrm{GeV}]$} \\
\hline & & & & & $m_{\tilde{g}}$ & $m_{\tilde{u}_{1}}$ & $m_{\tilde{d}_{1}}$ & $m_{\tilde{\ell}_{1}}$ & $m_{\tilde{\nu}_{1}}$ & $m_{\tilde{\chi}_{1}^{0}}$ & $m_{\tilde{\chi}_{2}^{0}}$ & $m_{\tilde{\chi}_{1}^{ \pm}}$ & $m_{h^{0}}$ \\
\hline SPS9 & 60 & 0.45 & 10 & + & 1321.5 & 950.0 & 1133.3 & 339.7 & 364.1 & 173.5 & 542.2 & 173.7 & 118.4 \\
\hline I & 60 & 1 & 10 & + & 1352.3 & 1087.6 & 1332.9 & 947.8 & 957.5 & 174.5 & 547.2 & 174.7 & 116.9 \\
\hline II & 60 & 2 & 20 & + & 1394.2 & 1469.7 & 1865.4 & 1906.4 & 1940.6 & 176.4 & 550.8 & 176.6 & 118.7 \\
\hline III & 60 & 3 & 30 & + & 1417.0 & 1950.7 & 2460.7 & 2743.1 & 2860.6 & 176.9 & 552.6 & 177.1 & 121.0 \\
\hline
\end{tabular}

differs very little from the SPS9 scenario, with a moderately larger universal scalar mass $m_{0}=1 \mathrm{TeV}$. As a consequence, the slepton masses are of about $1 \mathrm{TeV}$, while the squarks are only slightly heavier. Even if the lightest Higgs-boson mass is now a bit smaller, it lies still well above the excluded limit.

The points II and III feature higher values for $\tan \beta=20$ and 30 , respectively. In order to be able to solve the renormalization-group equations, one must then either decrease the value of $m_{3 / 2}$ or increase the value of $m_{0}$, as it can be seen from Fig. 1. Since the first possibility does not allow for large NMFV mixing in the squark sector due to the $b \rightarrow s \gamma$ branching ratio constraint, we adopt the second choice and fix the universal scalar mass to $m_{0}=2 \mathrm{TeV}$ and $3 \mathrm{TeV}$ for the scenarios II and III, respectively. Sfermions are therefore considerably heavier, with masses lying in the 2-3 TeV range. The mass of the lightest Higgs boson lies still above the constraint of Eq. (3.8). Let us note that for these two scenarios, the squarks are lighter than the sleptons, which may lead to nonleptonic supersymmetric cascade decays as a typical collider signature.

Starting from the minimally flavor-violating scenarios I, II, and III, we now include possible nonminimal flavor violation in the squark sector, i.e., we allow for $\lambda_{\mathrm{L}}, \lambda_{u}$ and $\lambda_{d}$ to be nonvanishing. However, we restrain ourselves to the cases where the off-diagonal entries in the squark mass matrices do not exceed the diagonal ones, i.e., $|\lambda|<$ 1. We investigate in Fig. 4 the dependence of the predictions for the observables $\operatorname{BR}(b \rightarrow s \gamma), \Delta M_{B_{s}^{0}}, \operatorname{BR}(b \rightarrow$ $s \mu \mu)$ and $\operatorname{BR}\left(B_{s}^{0} \rightarrow \mu^{+} \mu^{-}\right)$on the NMFV parameters and derive the allowed ranges for $\lambda_{\mathrm{L}}, \lambda_{u}$, and $\lambda_{d}$ under the assumption of a single nonvanishing parameter. We present in the $\left(\lambda_{\mathrm{L}}, \lambda_{u}\right)$ (upper panel) and $\left(\lambda_{\mathrm{L}}, \lambda_{d}\right)$ (lower panel) planes the regions excluded after confronting the AMSB predictions to the observed experimental values. The regions where the mass matrices, at the one-loop level, cannot be diagonalized are indicated in grey.

As can be seen, strong constraints are imposed by the decay $b \rightarrow s \gamma$ and the meson mass difference $\Delta M_{B_{s}^{0}}$. For all scenarios, while the $b \rightarrow s \gamma$ branching ratio data forbid too large (absolute) values for the $\lambda_{\mathrm{L}}$ parameter, $B_{s}^{0}-\bar{B}_{s}^{0}$ oscillations almost completely forbid negative values. Contrary, in the right-right chiral sector nonminimal flavor violation is left rather unconstrained, if not too large values of $\lambda_{\mathrm{L}}$ are assumed. Hence, in the case of the point I and assuming a single dominant $\lambda$-parameter hypothesis, i.e., allowing only one single nonvanishing off-diagonal element in the squark mass matrices, NMFV in the left-left chiral sector is constrained to $-0.22 \lesssim \lambda_{\mathrm{L}} \lesssim 0.42$ (with the two other parameters being set to $\lambda_{u}=\lambda_{d}=0$ ). On the other hand, assuming nonminimal flavor violation only in the right-right chiral sector $\left(\lambda_{\mathrm{L}}=0\right)$, we observe that both the $\lambda_{u}$ and $\lambda_{d}$ parameters are left almost unconstrained, leading to possible large flavor-violating effects.

Similarly, regarding scenario II and accounting for all constraints, the left-left chiral sector NMFV parameter is restricted to $-0.12 \lesssim \lambda_{\mathrm{L}} \lesssim 0.3$ in the $\lambda_{u}=\lambda_{d}=0$ case, and $\lambda_{u}$ is left unconstrained in the $\lambda_{\mathrm{L}}=0$ case. However, the higher value of $\tan \beta=20$ renders the $\Delta M_{B_{s}^{0}}$ observable sensitive to very high (absolute) values of $\lambda_{d}$, due to enhanced Yukawa couplings with the down-type (s)quark sector. This yields the constraint $-0.92 \lesssim \lambda_{d} \lesssim 0.96$ if the flavor violation is assumed to be located only in the downtype squark right-right chiral sector.

This effect on $\Delta M_{B_{s}^{0}}$ is a bit more pronounced for the third scenario, with its large value of $\tan \beta=30$, yielding moderate constraints for scenarios with nonminimal flavor violation in the right-right down-type squark chiral sector $\left(-0.88 \lesssim \lambda_{d} \lesssim 0.9\right)$. For NMFV in the left-left chiral sector, with $\lambda_{u}=\lambda_{d}=0$, one observes that the allowed range for $\lambda_{\mathrm{L}}$ is now severely restricted, as for the second scenario, to $-0.12 \lesssim \lambda_{\mathrm{L}} \lesssim 0.26$. All the results are summarized in Table II.

For scenario I, we show in Fig. 5 the dependence of the mass eigenvalues and the flavor decomposition of selected down-type squarks on the NMFV parameter $\lambda_{d}$, as an example, which induces a $\tilde{b}_{R}-\tilde{s}_{R}$ mixing. With increasing off-diagonal elements in the squark mass matrix, the resulting splitting of the physical mass eigenvalues becomes more important. Consequently, the lightest down-type squark $\tilde{d}_{1}$ becomes lighter, while the mass of the heaviest down-type state $\tilde{d}_{6}$ increases. 

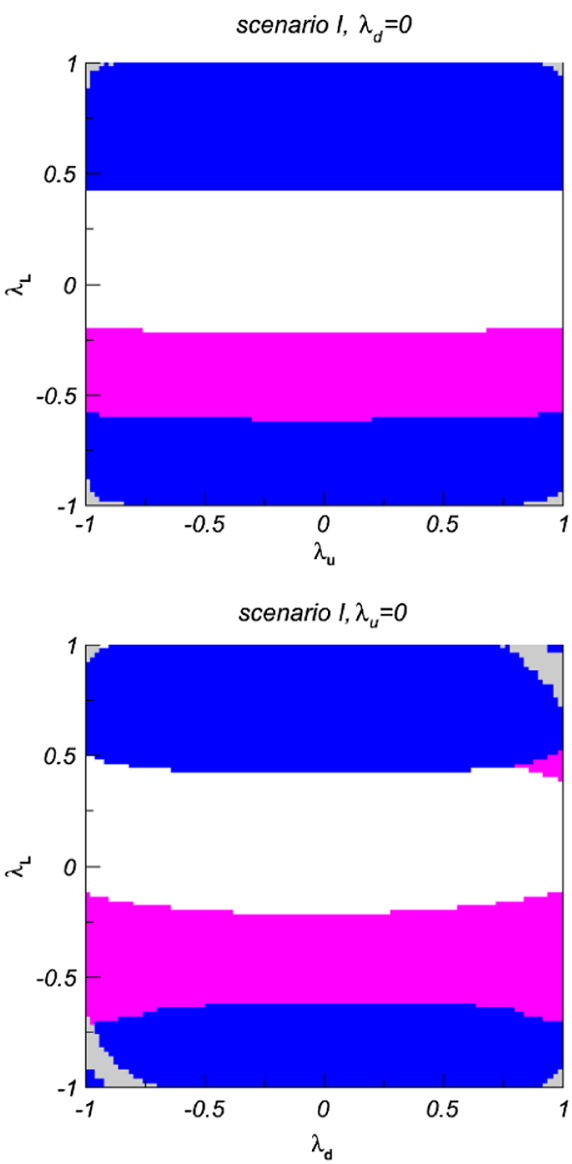

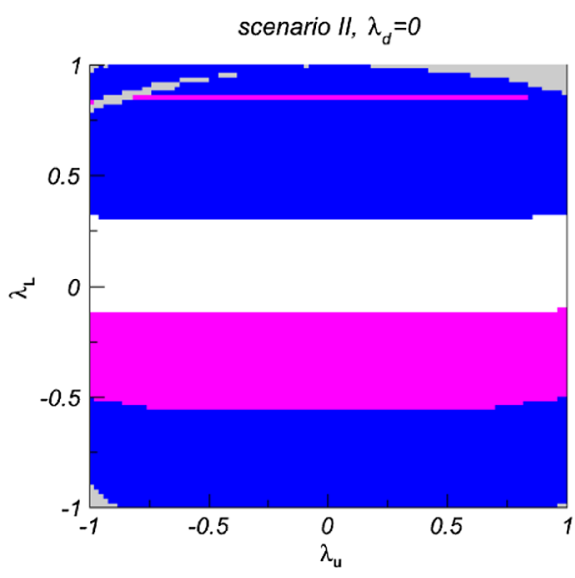

scenario $I I, \lambda_{d}=0$

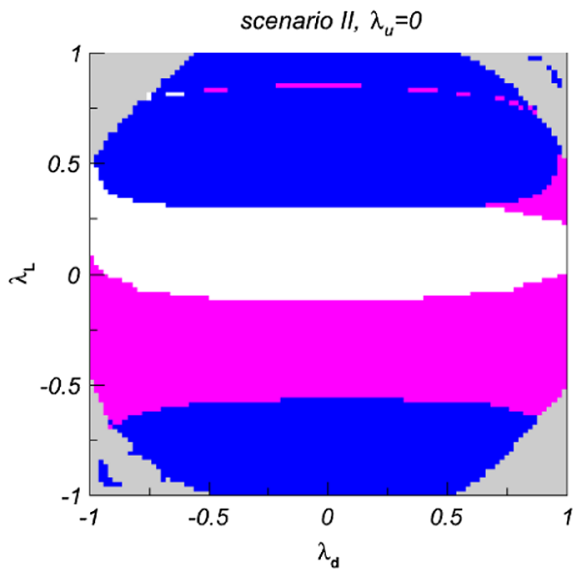

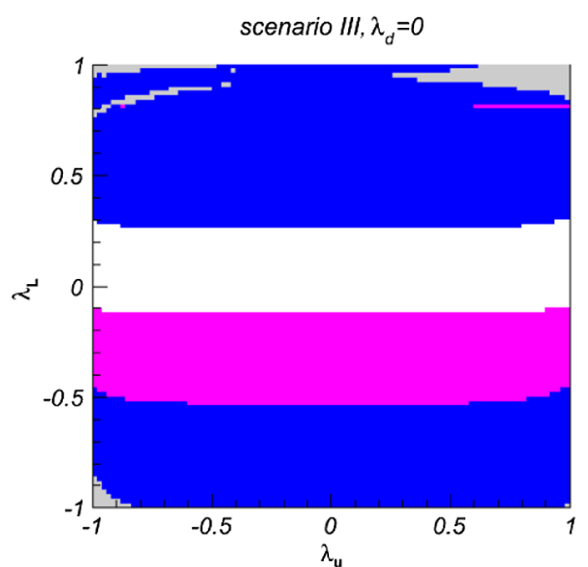

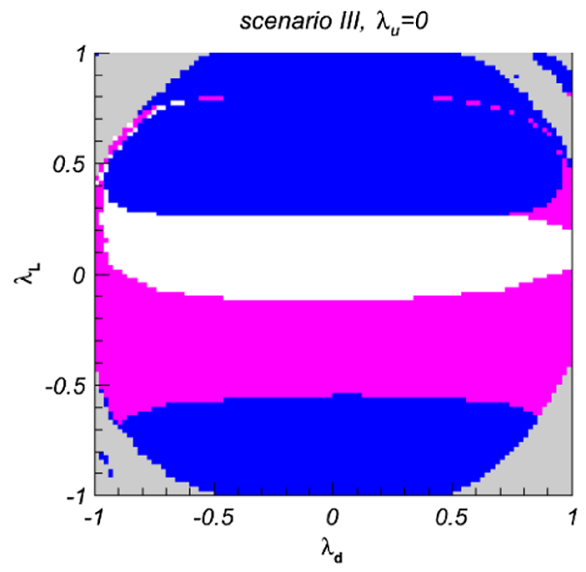

FIG. 4 (color online). $\quad\left(\lambda_{\mathrm{L}}, \lambda_{u}\right)$ (upper panel) and $\left(\lambda_{\mathrm{L}}, \lambda_{d}\right)$ (lower panel) planes for the three scenarios I (left), II (center) and III (right) presented in Table I. We show regions excluded due to the absence of physical solutions for the diagonalization of the mass matrices at the one-loop level (grey), the constraints associated to the $b \rightarrow s \gamma$ branching ratio (blue) and the $B$-meson mass difference $\Delta M_{B_{s}^{0}}$ (purple).

The altered mass splitting is accompanied by changes in the flavor decomposition of the involved squark states. In particular, at points where two squark mass eigenvalues should cross, the two corresponding eigenstates exchange their flavor content and undergo a so-called "avoided crossing" [4]. As can be seen in Fig. 5, in the MFV case the lightest down-type squark $\tilde{d}_{1}$ is a pure left-handed sbottom. For $\lambda_{d} \gtrsim 0.3$, this state acquires a sizeable admixture of the $\tilde{s}_{R}$ and $\tilde{b}_{R}$ squark, while its $\tilde{b}_{L}$ content drops accordingly. In a similar fashion, the next-to-lightest down-type squark $\tilde{d}_{2}$ is purely bottomlike at $\lambda_{d}=0$ and gets a sizeable strange admixture in the flavor-violating case. At $\lambda_{d} \sim 0.3$, an avoided crossing of the two mass eigenvalues of $\tilde{d}_{1}$ and $\tilde{d}_{2}$ is observed and the two states exchange their flavor content. For $\lambda_{d}>0.3$, the lightest down-type squark is then a mixture of the $\tilde{s}_{R}$ and $\tilde{b}_{R}$ eigenstates, while the next-to-lightest one becomes a pure $\tilde{b}_{L}$ state. Among the other mass eigenstates, only the heaviest down-type squark is fairly affected by $\lambda_{d}$ mixings. In the absence of additional flavour violation, i.e., in the $\lambda_{d}=0$ case, this state is purely $\tilde{d}_{R}$-like (not shown in the corresponding graph for the sake of visibility). However, for $\lambda_{d} \gtrsim 0$, it immediately undergoes an avoided crossing with $\tilde{d}_{5}$ and exchanges its flavor content, being then a pure $\tilde{s}_{R}$ state. With increasing values of the $\lambda_{d}$ parameter, this squark gradually gets a larger and larger $\tilde{b}_{R}$ component, and maximal mixing is reached for $\lambda_{d} \gtrsim 0.4$.

TABLE II. Ranges for the flavor-violating parameters $\lambda_{\mathrm{L}}, \lambda_{u}$ and $\lambda_{d}$ compatible with the low-energy and electroweak precision observables in the case of our three reference scenarios of Table I. The limits are given under the assumption of a single NMFV parameter, i.e., where only one single parameter is allowed to vary, the other being set to zero. If no value is indicated, the whole explored range of $-1<\lambda_{u, d}<1$ is allowed.

\begin{tabular}{l|ccc}
\hline \hline & $\lambda_{L}$ & $\lambda_{u}$ & $\lambda_{d}$ \\
\hline I & {$[-0.22,0.42]$} & $\cdots$ & $\cdots$ \\
II & {$[-0.12,0.30]$} & $\ldots$ & {$[-0.92,0.96]$} \\
III & {$[-0.12,0.26]$} & $\cdots$ & {$[-0.88,0.90]$} \\
\hline \hline
\end{tabular}



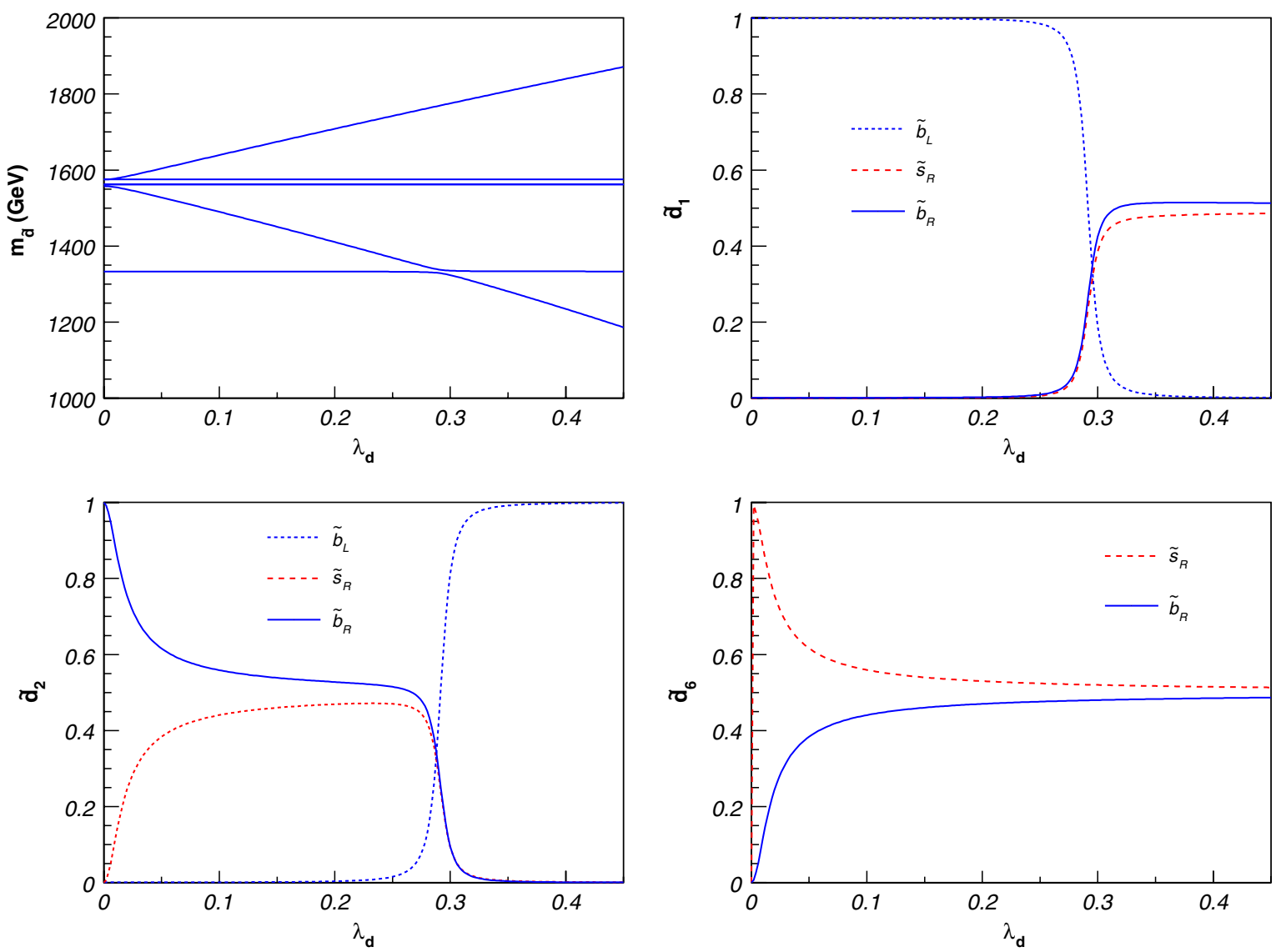

FIG. 5 (color online). Dependence of masses and flavor decomposition of the lightest, second lightest, and heaviest down-type squarks on the flavor-violating parameter $\lambda_{d}$ for the scenario I of Table I.

Avoided crossings and similar mass splittings also occur for the two other scenarios, as presented in Figs. 6 and 7, as well as for up-type squarks and the variations of the other flavorviolating parameters $\lambda_{\mathrm{L}}$ and $\lambda_{u}$ (not shown). This behavior can lead to interesting phenomenological consequences for production and decays of squarks and gluinos, as it has been shown in Refs. [4,5,56-61]. In particular, the dependence of the gluino production on the nonminimal flavor-violation parameters is discussed in the following section.

In Figs. 8-10, we show the theoretical predictions for the branching ratios of the rare decays $b \rightarrow s \gamma$ and $b \rightarrow s \mu \mu$ as well as the meson-oscillation observable $\Delta M_{B_{s}^{0}}$ as a function of the left-left mixing parameter $\lambda_{L}$. These three observables are rather sensitive to nonminimal flavor violation and can therefore be used to constrain the possible values of the $\lambda$ parameters, contrary to the branching ratio $B_{s}^{0} \rightarrow \mu^{+} \mu^{-}$, for which the predictions lie well below the observed upper bound of Eq. (3.4), for any value for the $\lambda$ parameters, and are given by $(5.0 \ldots 5.2) \times 10^{-9}$, $(5.2 \ldots 6.3) \times 10^{-9}$ and $(5.0 \ldots 9.0) \times 10^{-9}$ for our scenarios I, II and III, respectively. As already discussed above, the decay $b \rightarrow s \gamma$ is very sensitive to additional flavour mixing, particularly in the left-left sector. Together with its high experimental precision, this makes it the most stringent constraint in this case. Hence, as shown in the figures, the lower limit of the interval given in Eq. (3.2) is reached (at the $2 \sigma$ confidence level) at $\lambda_{L} \sim 0.4$, according to the corresponding values derived in Table II. The prediction of $\Delta M_{B_{s}^{0}}$ also strongly depends on the mixing in the left-left chiral sector. The lower bound of Eq. (3.5) is reached at $\lambda_{\mathrm{L}} \sim 0.43$, making this constraint competitive to the rare decay $b \rightarrow s \gamma$ despite the large theoretical uncertainty. Finally, constraints from the $b \rightarrow s \mu \mu$ observable are weaker for values of the mixing parameter $\lambda_{L} \lesssim 0.6$, the theoretical predictions showing no significant dependence on flavor mixing in that $\lambda_{\mathrm{L}}$ region.

\section{NONMINIMALLY FLAVOR-VIOLATING GLUINO HADROPRODUCTION}

\section{A. Generalized couplings in NMFV supersymmetry}

In our previous publications, we have computed the cross sections for the production of gaugino and squark pairs in the framework of nonminimally flavor-violating supersymmetry [4,5]. In this work, we add the missing channels related to gluino production. We first introduce our conventions for the generalized strong and electroweak couplings which will appear in our analytical calculations 

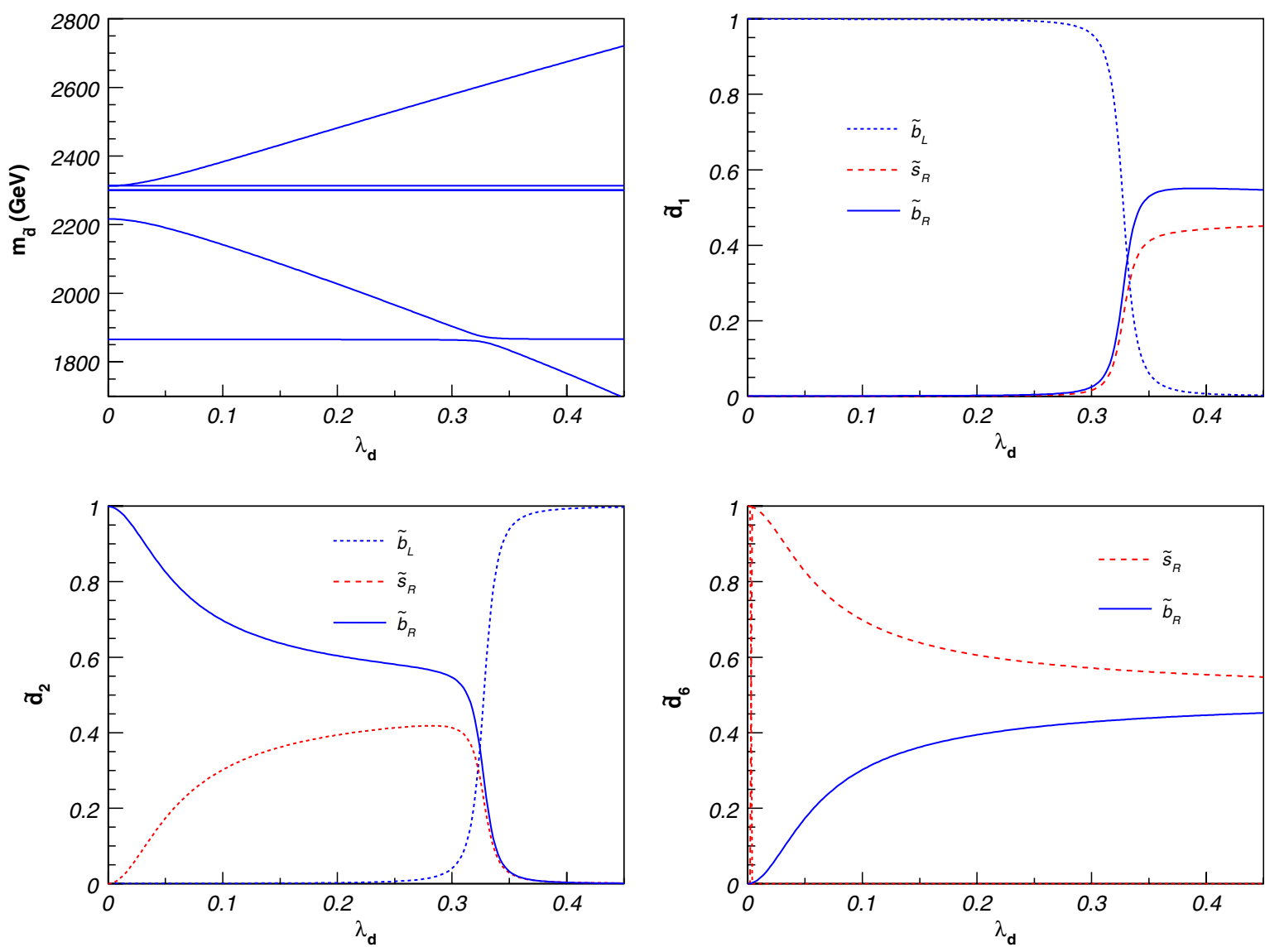

FIG. 6 (color online). Same as Fig. 5 for the scenario II of Table I.

of the cross sections. Nonminimal flavor violation in the strong sector can arise from interactions between gluinos $\tilde{g}$, quarks $q$ and left- (right-)handed squarks $\tilde{q}_{L}\left(\tilde{q}_{R}\right)$ described by the (flavor-diagonal) Lagrangian $[1,2]$

$$
\mathcal{L}_{q \tilde{q} \tilde{g}}=\sqrt{2} g_{s}\left[-\tilde{q}_{L f}^{\dagger} T_{a}\left(\tilde{g}^{a} P_{L} \bar{q}_{f}\right)+\left(\bar{q}_{f} P_{L} \tilde{g}^{a}\right) T_{a} \tilde{q}_{R f}\right]+\text { H.c., }
$$

where $f$ stands for a flavor index, $T_{a}$ and $g_{s}$ are the fundamental matrices and the coupling constant associated with the $S U(3)_{c}$ gauge group, and $P_{L}$ denotes the leftchirality projection operator acting on four-component spinors. Rotating to the mass-eigenstate basis, flavor violation is introduced through possible nondiagonal entries in the matrices $R^{q}$. In this case, the left-handed and righthanded coupling strengths become proportional to

$$
\left\{L_{\tilde{q}_{i} q_{j} \tilde{g}}, R_{\tilde{q}_{i} q_{j} \tilde{g}}\right\}=\left\{R_{i j}^{q},-R_{i(j+3)}^{q}\right\} .
$$

Similarly, for the electroweak sector, nonminimally flavorviolating interactions can arise in the chargino and neutralino couplings to quarks and squarks. We start from the Lagrangian

$$
\begin{aligned}
\mathcal{L}_{q \tilde{q} \tilde{\chi}}= & \sqrt{2} g^{\prime}\left[-\frac{1}{6} \tilde{q}_{L f}^{\dagger}\left(\overline{\tilde{B}} P_{L} q_{f}\right)+\frac{2}{3}\left(\bar{u}_{f} P_{L} \tilde{B}\right) \tilde{u}_{R f}\right. \\
& \left.-\frac{1}{3}\left(\bar{d}_{f} P_{L} \tilde{B}\right) \tilde{d}_{R f}\right]-\frac{\sqrt{2}}{2} g_{W} \tilde{Q}_{f}^{\dagger} \sigma_{k}\left(\overline{\tilde{W}}^{k} P_{L} Q_{f}\right) \\
& -\left(y_{d}\right)_{f f^{\prime}} \tilde{d}_{f}^{\dagger} \overline{\tilde{H}}_{d} P_{L} Q_{f^{\prime}}+\left(y_{d}\right)_{f f^{\prime}} \tilde{Q}_{f^{\prime}} \bar{d}_{f} P_{L} \tilde{H}_{d} \\
& +\left(y_{u}\right)_{f f^{\prime}} \tilde{u}_{f}^{\dagger} \overline{\tilde{H}}_{u} P_{L} Q_{f^{\prime}}-\left(y_{u}\right)_{f f^{\prime}} \tilde{Q}_{f^{\prime}} \bar{u}_{f} P_{L} \tilde{H}_{u}+\text { H.c., }
\end{aligned}
$$

where $\tilde{B}, \tilde{W}$ and $\tilde{H}_{\{u, d\}}$ are the four-component bino, wino and Higgsino eigenstates and $Q(\tilde{Q}), u(\tilde{u})$ and $d(\tilde{d})$ the doublet of left-handed (s)quarks and the up- and down-type right-handed (s)quarks, respectively. In the Lagrangian above, we denote the hypercharge and weak coupling constants $g^{\prime}$ and $g_{W}$, while the generators of $S U(2)_{L}$ are given by $\sigma / 2, \sigma$ being the Pauli matrices. Finally, $y_{u}$ and $y_{d}$ are the up-type and down-type quark Yukawa matrices, which once diagonalized are proportional to the quark masses,

$$
\left(\hat{y}_{u}\right)_{i j}=\frac{\sqrt{2} g_{W} m_{u_{i}}}{2 m_{W} \sin \beta} \delta_{i j} \quad \text { and } \quad\left(\hat{y}_{d}\right)_{i j}=\frac{\sqrt{2} g_{W} m_{d_{i}}}{2 m_{W} \cos \beta} \delta_{i j} .
$$

Here, $\hat{y}_{\{u, d\}}$ denote the diagonalized Yukawa matrices. After rotating to the mass basis, one obtains the coupling strengths 


$$
\begin{aligned}
& L_{\tilde{d}_{j} d_{k} \tilde{\chi}_{i}^{0}}=\frac{\left(e_{d}-T_{d}^{3}\right) s_{W} N_{i 1}+T_{d}^{3} c_{W} N_{i 2}}{\sqrt{2} c_{W}} R_{j k}^{d *}+\frac{m_{d_{k}} N_{i 3}}{2 \sqrt{2} m_{W} \cos \beta} R_{j(k+3)}^{d *}, \\
& R_{\tilde{d}_{j} d_{k} \tilde{\chi}_{i}^{0}}=-\frac{e_{d} s_{W} N_{i 1}^{*}}{\sqrt{2} c_{W}} R_{j(k+3)}^{d^{*}}+\frac{m_{d_{k}} N_{i 3}^{*}}{2 \sqrt{2} m_{W} \cos \beta} R_{j k}^{d^{*}}, \\
& L_{\tilde{u}_{j} u_{k} \tilde{\chi}_{i}^{0}}=\frac{\left(e_{u}-T_{u}^{3}\right) s_{W} N_{i 1}+T_{u}^{3} c_{W} N_{i 2}}{\sqrt{2} c_{W}} R_{j k}^{u^{*}}+\frac{m_{u_{k}} N_{i 4}}{2 \sqrt{2} m_{W} \sin \beta} R_{j(k+3)}^{u^{*}}, \\
& R_{\tilde{u}_{j} u_{k} \tilde{\chi}_{i}^{0}}=-\frac{e_{u} s_{W} N_{i 1}^{*}}{\sqrt{2} c_{W}} R_{j(k+3)}^{u^{*}}+\frac{m_{u_{k}} N_{i 4}^{*}}{2 \sqrt{2} m_{W} \sin \beta} R_{j k}^{u^{*}}, \\
& L_{\tilde{d}_{j} u_{k} \tilde{\chi}_{i}^{ \pm}}=\frac{1}{2} \sum_{l=1}^{3}\left[U_{i 1} R_{j l}^{d *}-\frac{m_{d_{l}} U_{i 2}}{\sqrt{2} m_{W} \cos \beta} R_{j(l+3)}^{d *}\right] V_{u_{k} d_{l}}, \\
& R_{\tilde{d}_{j} u_{k} \tilde{\chi}_{i}^{ \pm}}=-\sum_{l=1}^{3} \frac{m_{u_{k}} V_{i 2}^{*} V_{u_{k} d_{l}}}{2 \sqrt{2} m_{W} \sin \beta} R_{j l}^{d *}, \\
& L_{\tilde{u}_{j} d_{k} \tilde{\chi}_{i}^{ \pm}}=\frac{1}{2} \sum_{l=1}^{3}\left[V_{i 1} R_{j l}^{u *}-\frac{m_{u_{l}} V_{i 2}}{\sqrt{2} m_{W} \sin \beta} R_{j(l+3)}^{u^{*}}\right] V_{u_{l} d_{k}}^{*} \text {, } \\
& R_{\tilde{u}_{j} d_{k} \tilde{x}_{i}^{ \pm}}=-\sum_{l=1}^{3} \frac{m_{d_{k}} U_{i 2}^{*} V_{u_{l} d_{k}}^{*}}{2 \sqrt{2} m_{W} \cos \beta} R_{j l}^{u *},
\end{aligned}
$$
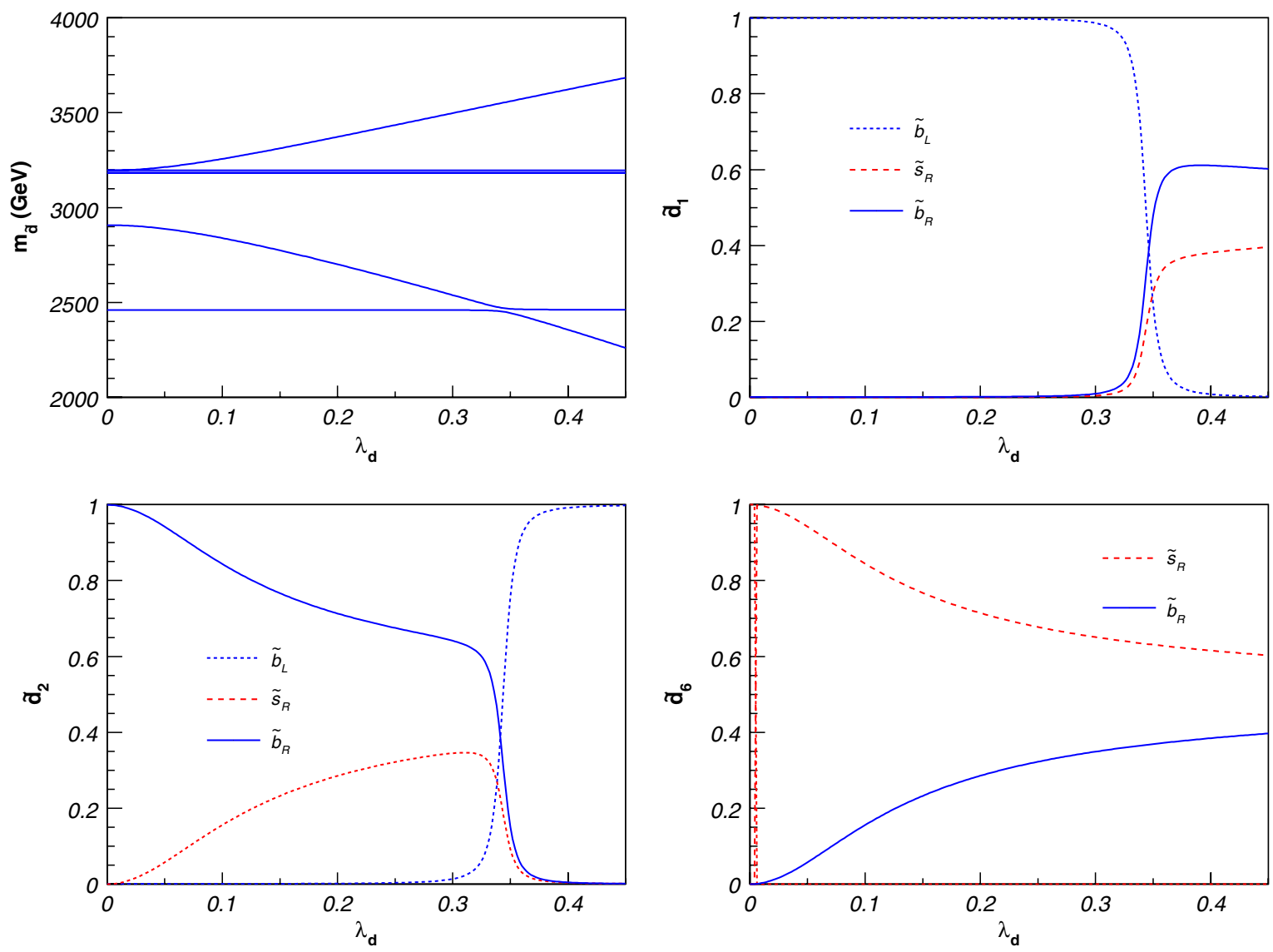

FIG. 7 (color online). Same as Fig. 5 for the scenario III of Table I. 

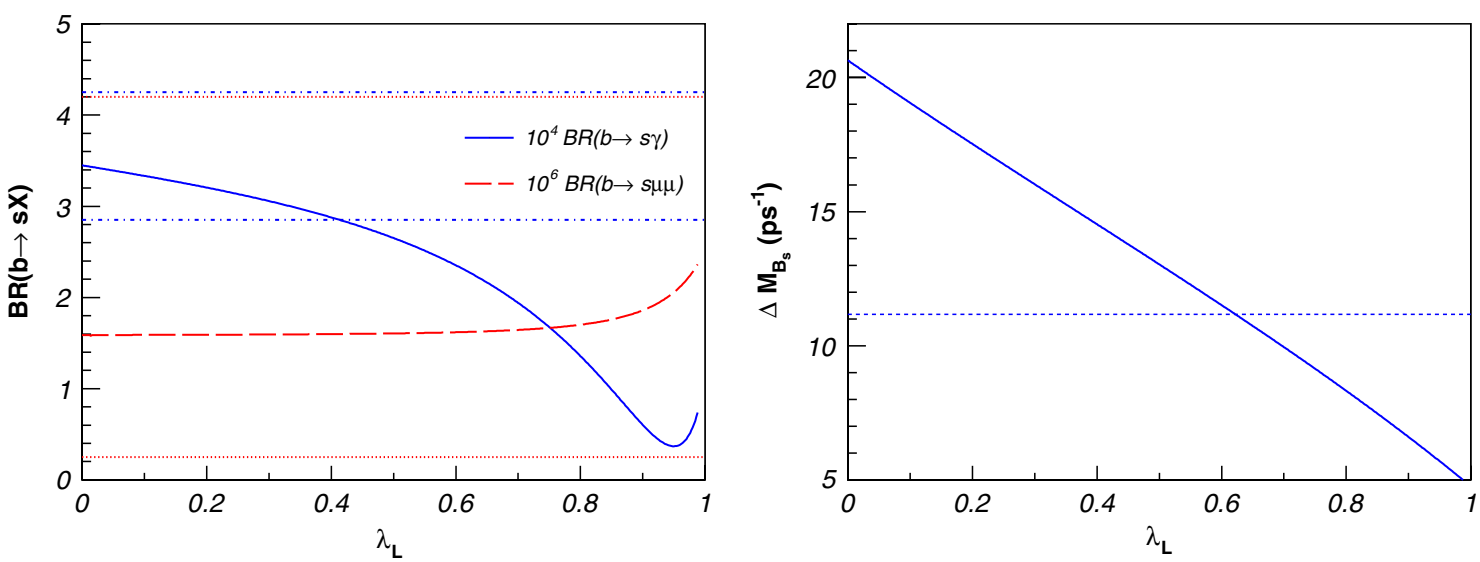

FIG. 8 (color online). Dependence of $\mathrm{BR}(b \rightarrow s \gamma), \mathrm{BR}(b \rightarrow s \mu \mu)$, and $\Delta M_{B_{s}^{0}}$ on the flavor-violating parameter $\lambda_{L}$ for the scenario I of Table I. We also show horizontal lines corresponding to the experimental upper and lower limits for $\mathrm{BR}(b \rightarrow s \gamma)$ (blue dashed line), $\mathrm{BR}(b \rightarrow s \mu \mu)$ (red dotted line), and $\Delta M_{B_{s}^{0}}$ (blue dashed line), as discussed in Sec. III A.
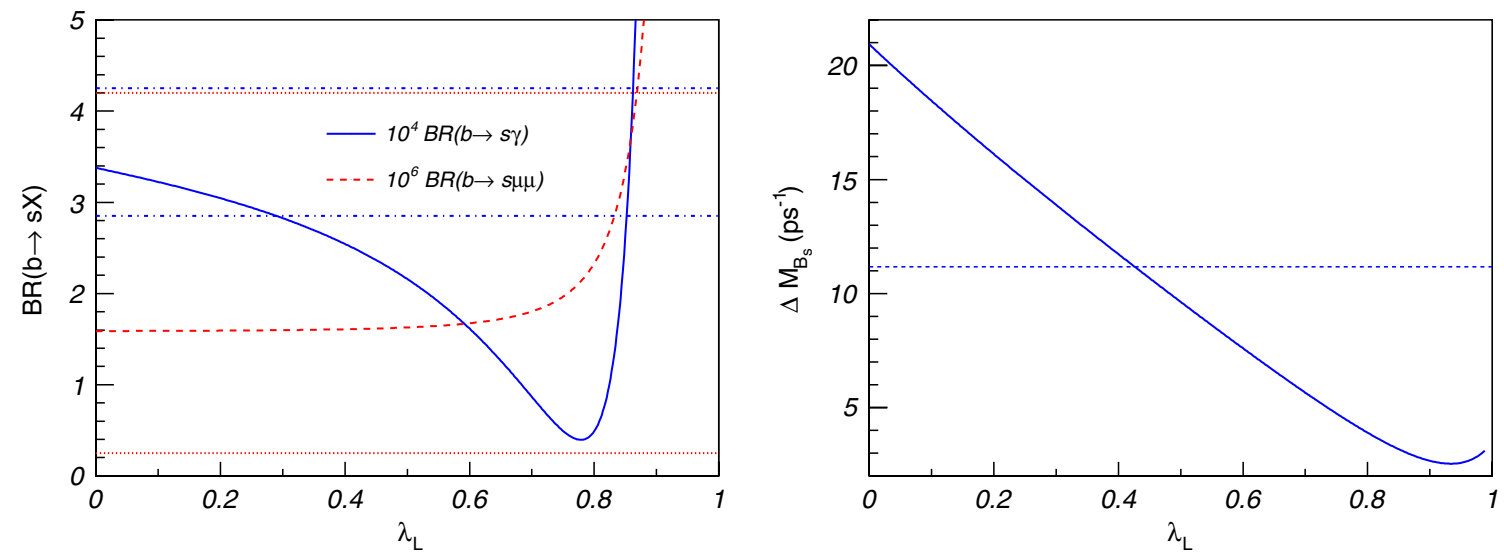

FIG. 9 (color online). Same as Fig. 8 for the scenario II of Table I.
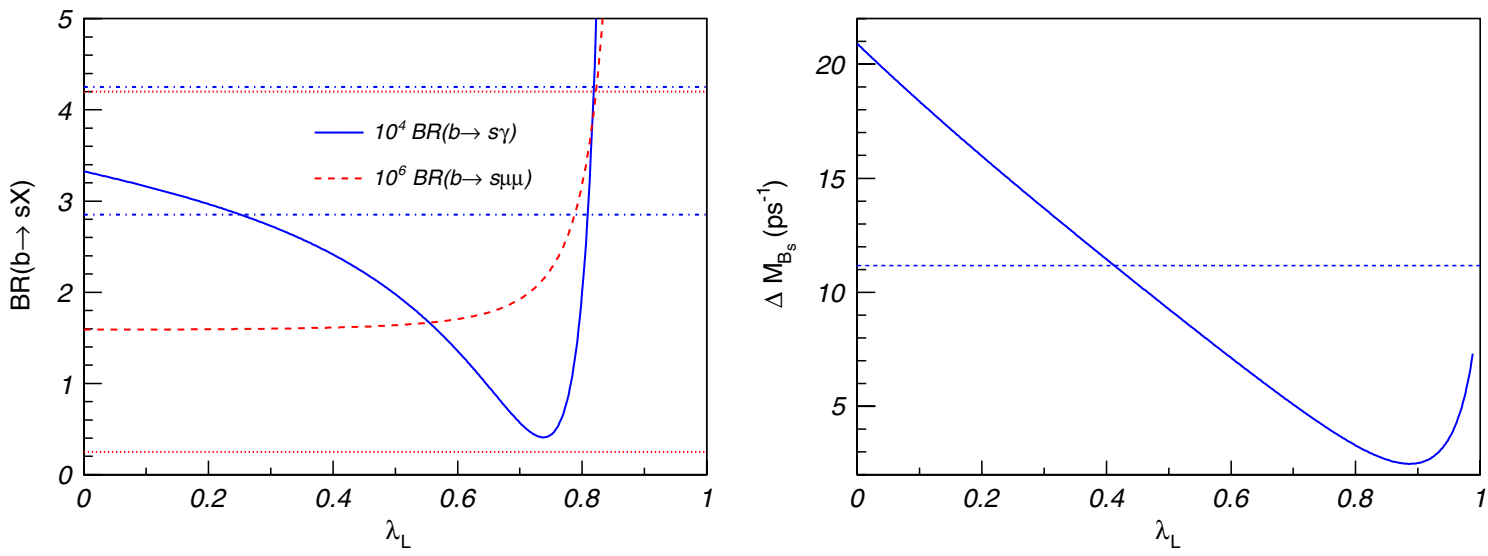

FIG. 10 (color online). Same as Fig. 8 for the scenario III of Table I. 
where we follow the notations of Sec. II. In addition, we introduce the mass of the $W$ boson $m_{W}$, the cosine of the electroweak mixing angle $c_{W}$, and the matrices $N, U$ and $V$ related to the gaugino/Higgsino mixing.

\section{B. Analytical results}

We compute the partonic cross sections related to gluino production, i.e., for the processes

$$
\begin{aligned}
& a_{h_{a}}\left(p_{a}\right) b_{h_{b}}\left(p_{b}\right) \rightarrow \tilde{g}\left(p_{1}\right) \tilde{q}_{i}^{(*)}\left(p_{2}\right), \\
& \tilde{g}\left(p_{1}\right) \tilde{g}\left(p_{2}\right) \quad \text { and } \quad \tilde{g}\left(p_{1}\right) \tilde{\chi}_{i}^{\{ \pm, 0\}}\left(p_{2}\right),
\end{aligned}
$$

and present the results for definite helicities $h_{a, b}$ of the initial partons $a, b=q, \bar{q}, g$ in terms of the squark masses $m_{\tilde{q}_{j}}$, the chargino and neutralino masses $m_{\tilde{\chi}_{j}}$, the gluino mass $m_{\tilde{g}}$, the Mandelstam variables

$$
\begin{aligned}
s & =\left(p_{a}+p_{b}\right)^{2}, \\
t & =\left(p_{a}-p_{1}\right)^{2}, \quad \text { and } \\
u & =\left(p_{a}-p_{2}\right)^{2},
\end{aligned}
$$

and the mass-subtracted Mandelstam variables

$$
\begin{gathered}
t_{\tilde{g}}=t-m_{\tilde{g}}^{2}, \quad u_{\tilde{g}}=u-m_{\tilde{g}}^{2}, \quad t_{\tilde{q}_{i}}=t-m_{\tilde{q}_{i}}^{2}, \\
u_{\tilde{q}_{i}}=u-m_{\tilde{q}_{i}}^{2}, \quad t_{\tilde{\chi}_{i}}=t-m_{\tilde{\chi}_{i}}^{2}, \quad u_{\tilde{\chi}_{i}}=u-m_{\tilde{\chi}_{i}}^{2} .
\end{gathered}
$$

Unpolarized partonic cross sections $d \hat{\sigma}$ and single- and double-polarized cross partonic sections $d \hat{\sigma}_{L}$ and $d \hat{\sigma}_{L L}$, averaged over initial spins, can easily be derived from the helicity-dependent result,

$$
\begin{aligned}
\mathrm{d} \hat{\sigma} & =\frac{\mathrm{d} \hat{\sigma}_{1,1}+\mathrm{d} \hat{\sigma}_{1,-1}+\mathrm{d} \hat{\sigma}_{-1,1}+\mathrm{d} \hat{\sigma}_{-1,-1}}{4}, \\
\mathrm{~d} \Delta \hat{\sigma}_{L} & =\frac{\mathrm{d} \hat{\sigma}_{1,1}+\mathrm{d} \hat{\sigma}_{1,-1}-\mathrm{d} \hat{\sigma}_{-1,1}-\mathrm{d} \hat{\sigma}_{-1,-1}}{4}, \\
\mathrm{~d} \Delta \hat{\sigma}_{L L} & =\frac{\mathrm{d} \hat{\sigma}_{1,1}-\mathrm{d} \hat{\sigma}_{1,-1}-\mathrm{d} \hat{\sigma}_{-1,1}+\mathrm{d} \hat{\sigma}_{-1,-1}}{4} .
\end{aligned}
$$

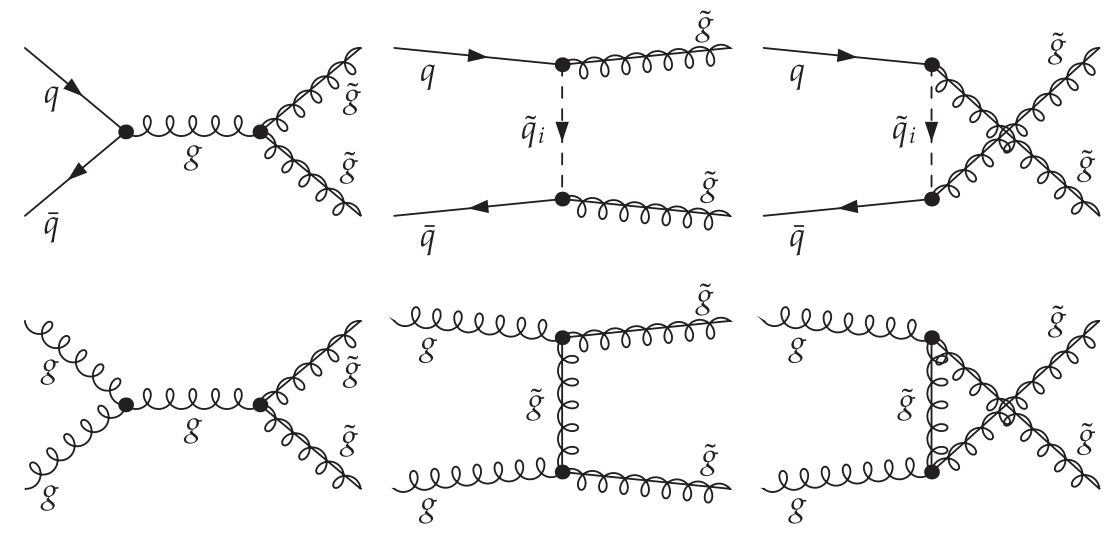

FIG. 11. Tree-level Feynman diagrams for the production of a pair of gluinos in quark-antiquark (top) and gluon-gluon collisions (bottom). 


$$
\begin{aligned}
\frac{\mathrm{d} \hat{\sigma}}{\mathrm{d} t}\left(h_{a}, h_{b}\right)= & \frac{g_{s}^{4}}{128 \pi s^{2} N_{c}^{2}}\left[\left(1-h_{a}\right)\left(1+h_{b}\right)\left[\frac{Q_{s s}}{s^{2}}+\sum_{i=1}^{6}\left\{\frac{\left(Q_{s t}\right)_{i}^{1}}{s t_{\tilde{q}_{i}}}+\frac{\left(Q_{s u}\right)_{i}^{1}}{s u_{\tilde{q}_{i}}}\right\}+\sum_{i, j=1}^{6}\left\{\frac{\left(Q_{t t}\right)_{i j}^{11}}{t_{\tilde{q}_{i}} t_{\tilde{q}_{j}}}+\frac{\left(Q_{u u}\right)_{i j}^{11}}{u_{\tilde{q}_{i}} u_{\tilde{q}_{j}}}+\frac{\left(Q_{t u}\right)_{i j}^{11}}{t_{\tilde{q}_{i}} u_{\tilde{q}_{j}}}\right\}\right]\right. \\
& +\left(1+h_{a}\right)\left(1-h_{b}\right)\left[\frac{Q_{s s}}{s^{2}}+\sum_{i=1}^{6}\left\{\frac{\left(Q_{s t}\right)_{i}^{2}}{s t_{\tilde{q}_{i}}}+\frac{\left(Q_{s u}\right)_{i}^{2}}{s u_{\tilde{q}_{i}}}\right\}+\sum_{i, j=1}^{6}\left\{\frac{\left(Q_{t t}\right)_{i j}^{22}}{t_{\tilde{q}_{i}} t_{\tilde{q}_{j}}}+\frac{\left(Q_{u u}\right)_{i j}^{22}}{u_{\tilde{q}_{i}} u_{\tilde{q}_{j}}}+\frac{\left(Q_{t u}\right)_{i j}^{22}}{t_{\tilde{q}_{i}} u_{\tilde{q}_{j}}}\right\}\right] \\
& +\left(1-h_{a}\right)\left(1-h_{b}\right)\left[\sum_{i, j=1}^{6}\left\{\frac{\left(Q_{t t}\right)_{i j}^{12}}{t_{\tilde{q}_{i}} \tilde{q}_{\tilde{q}_{j}}}+\frac{\left(Q_{u u}\right)_{i j}^{12}}{u_{\tilde{q}_{i}} u_{\tilde{q}_{j}}}+\frac{\left(Q_{t u}\right)_{i j}}{t_{\tilde{q}_{i}} u_{\tilde{q}_{j}}}\right\}\right] \\
& \left.+\left(1+h_{a}\right)\left(1+h_{b}\right)\left[\sum_{i, j=1}^{6}\left\{\frac{\left(Q_{t t}\right)_{i j}^{21}}{t_{\tilde{q}_{i}} t_{\tilde{q}_{j}}}+\frac{\left(Q_{u u}\right)_{i j}^{21}}{u_{\tilde{q}_{i}} u_{\tilde{q}_{j}}}+\frac{\left(Q_{t u}\right)_{i j}^{21}}{t_{\tilde{q}_{i}} u_{\tilde{q}_{j}}}\right\}\right]\right]
\end{aligned}
$$

with the generalized charges

$$
\begin{aligned}
Q_{s s} & =2 N_{c}\left(N_{c}^{2}-1\right)\left(t_{\tilde{g}}^{2}+u_{\tilde{g}}^{2}+2 m_{\tilde{g}}^{2} s\right), \\
\left(Q_{s t}\right)_{i}^{m} & =N_{c}\left(N_{c}^{2}-1\right) \operatorname{Re}\left[\mathcal{C}_{\tilde{q}_{i} q \tilde{g}}^{m} \mathcal{C}_{\tilde{q}_{i} q^{\prime} \tilde{g}}^{m *}\right]\left(m_{\tilde{g}}^{2} s+t_{\tilde{g}}^{2}\right), \\
\left(Q_{s u}\right)_{i}^{m} & =N_{c}\left(N_{c}^{2}-1\right) \operatorname{Re}\left[\mathcal{C}_{\tilde{q}_{i} q \tilde{g}}^{m} \mathcal{C}_{\tilde{q}_{i} q^{\prime} \tilde{g}}^{m *}\right]\left(m_{\tilde{g}}^{2} s+u_{\tilde{g}}^{2}\right), \\
\left(Q_{t t}\right)_{i j}^{m n} & =\frac{\left(N_{c}^{2}-1\right)^{2}}{N_{c}} \mathcal{C}_{\tilde{q}_{i} q \tilde{g}}^{m} \mathcal{g}_{\tilde{q}_{i} q^{\prime} \tilde{g}}^{n *} \mathcal{C}_{\tilde{q}_{j} q \tilde{g}}^{m *} \mathcal{C}_{\tilde{q}_{j} q^{\prime} \tilde{g}}^{n} t_{\tilde{g}}^{2}, \\
\left(Q_{u u}\right)_{i j}^{m n} & =\frac{\left(N_{c}^{2}-1\right)^{2}}{N_{c}} \mathcal{C}_{\tilde{q}_{i} q \tilde{g}}^{m} \mathcal{C}_{\tilde{q}_{i} q^{\prime} \tilde{g}}^{n^{*}} \mathcal{C}_{\tilde{q}_{j} q \tilde{g}}^{m *} \mathcal{C}_{\tilde{q}_{j} q^{\prime} \tilde{g}}^{n} u_{\tilde{g}}^{2}, \\
\left(Q_{t u}\right)_{i j}^{m n} & =2 \frac{\left(N_{c}^{2}-1\right)}{N_{c}} \operatorname{Re}\left[\mathcal{C}_{\tilde{q}_{i} q \tilde{g}}^{m} \mathcal{C}_{\tilde{q}_{i} q^{\prime} \tilde{g}}^{n *} \mathcal{C}_{\tilde{q}_{j} q^{\prime} \tilde{g}}^{n} \mathcal{C}_{\tilde{q}_{j} q \tilde{g}}^{m *}\right]\left(\left(1-\delta_{m n}\right)\left(m_{\tilde{g}}^{2} s-t_{\tilde{g}} u_{\tilde{g}}\right)+\delta_{m n} m_{\tilde{g}}^{2} s\right),
\end{aligned}
$$

where for the sake of simplicity we have introduced the generic notation

$$
\left\{\mathcal{C}_{a b c}^{1}, \mathcal{C}_{a b c}^{2}\right\}=\left\{L_{a b c}, R_{a b c}\right\} .
$$

This reproduces both the polarized and unpolarized results of Refs. [62,63] in the flavor-conserving MSSM limit.

An associated pair of a gluino and a squark originates from quark-gluon initial states,

$$
q_{h_{a}}\left(p_{a}\right) g_{h_{b}}\left(p_{b}\right) \rightarrow \tilde{g}\left(p_{1}\right) \tilde{q}_{i}\left(p_{2}\right),
$$

and proceeds through an $s$-channel quark, $t$-channel squark, or $u$-channel gluino exchange as it is illustrated in Fig. 12. Since each contribution involves a coupling between a quark, a squark and a gluino, this process can in general violate flavor. The differential cross section is given by

$$
\begin{aligned}
\frac{\mathrm{d} \hat{\sigma}}{\mathrm{d} t}\left(h_{a}, h_{b}\right)= & \frac{g_{s}^{4}}{64 \pi s^{2}}\left\{\left[\left(1-h_{a}\right)\left(1-h_{b}\right)\left|L_{\tilde{q}_{i} q \tilde{g}}\right|^{2}+\left(1+h_{a}\right)\left(1+h_{b}\right)\left|R_{\tilde{q}_{i} q \tilde{g}}\right|^{2}\right]\right. \\
& \times\left[\frac{-\left(N_{c}^{2}-1\right) u_{\tilde{g}}}{4 N_{c}^{2} s}+\frac{s t+t_{\tilde{g}} t_{\tilde{q}_{i}}}{2 N_{c}^{2} s t_{\tilde{q}_{i}}}+\frac{1}{2 u_{\tilde{g}}}\left(\frac{2 s u-s u_{\tilde{g}}+2 u_{\tilde{q}_{i}} u_{\tilde{g}}}{u_{\tilde{g}}}+\frac{m_{\tilde{g}}^{2} s+u_{\tilde{g}} u_{\tilde{q}_{i}}}{s}+\frac{m_{\tilde{g}}^{2} m_{\tilde{q}_{i}}^{2}-t u}{t_{\tilde{q}_{i}}}\right)\right] \\
& \left.+\left[\left(1-h_{a}\right)\left|L_{\tilde{q}_{i} q \tilde{g}}\right|^{2}+\left(1+h_{a}\right)\left|R_{\tilde{q}_{i} q \tilde{g}}\right|^{2}\right] \times\left[\frac{\left(N_{c}^{2}-1\right) m_{\tilde{q}_{i}}^{2} t_{\tilde{g}}}{2 N_{c}^{2} t_{\tilde{q}_{i}}^{2}}-\frac{t_{\tilde{g}}\left(s+t_{\tilde{g}}\right)}{2 N_{c}^{2} s t_{\tilde{q}_{i}}} \frac{1}{2 u_{\tilde{g}}}\left(\frac{2 u t_{\tilde{g}}}{u_{\tilde{g}}}-\frac{u_{\tilde{q}_{i}}\left(s+u_{\tilde{q}_{i}}\right)}{s}+\frac{2 u-u_{\tilde{q}_{i}}}{t_{\tilde{q}_{i}}}\right)\right]\right\},
\end{aligned}
$$

which agrees again with the polarized and unpolarized results of Refs. [62,63] in the flavor-conserving MSSM limit and after summing over mass-degenerate squarks. The cross section for the charge-conjugate process can be easily derived by replacing $h_{a} \rightarrow-h_{a}$.

Finally, the associated production of a gluino and a chargino or of a gluino and a neutralino,

$$
q_{h_{a}}\left(p_{a}\right) \bar{q}_{h_{b}}\left(p_{b}\right) \rightarrow \tilde{g}\left(p_{1}\right) \tilde{\chi}_{i}^{ \pm, 0}\left(p_{2}\right)
$$

can be mediated through a $t$-channel or $u$-channel squark exchange, as shown in Fig. 13, and can thus involve flavorviolating interactions. The differential cross section is given by 

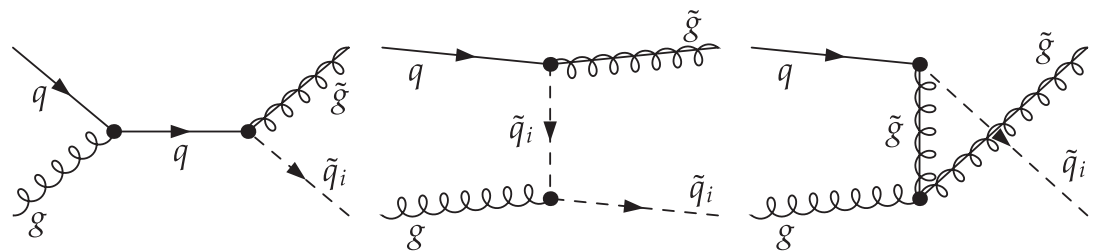

FIG. 12. Tree-level Feynman diagrams for the associated production of gluinos and squarks.

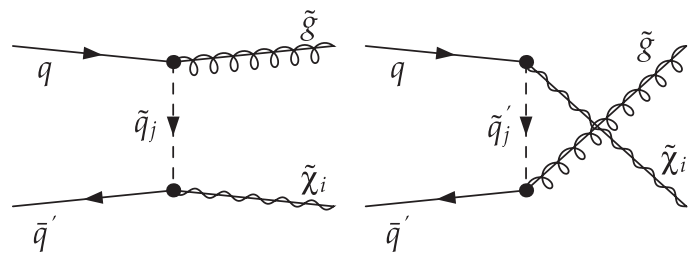

FIG. 13. Tree-level Feynman diagrams for the associated production of gluinos and gauginos.

$$
\begin{aligned}
\frac{\mathrm{d} \hat{\sigma}}{\mathrm{d} t}\left(h_{a}, h_{b}\right)= & \frac{g_{s}^{2} g_{W}^{2}\left(N_{c}^{2}-1\right)}{8 \pi s^{2} N_{c}^{2}} \sum_{i, j=1}^{6}\left\{\left(1-h_{a}\right)\left(1+h_{b}\right)\left[\frac{\left(Q_{t t}\right)_{i j}^{11}}{t_{\tilde{q}_{i}} t_{\tilde{q}_{j}}}+\frac{\left(Q_{u u}\right)_{i j}^{11}}{u_{\tilde{q}_{i}} u_{\tilde{q}_{j}}}+\frac{\left(Q_{t u}\right)_{i j}^{11}}{t_{\tilde{q}_{i}} u_{\tilde{q}_{j}}}\right]\right. \\
& +\left(1+h_{a}\right)\left(1-h_{b}\right)\left[\frac{\left(Q_{t t}\right)_{i j}^{22}}{t_{\tilde{q}_{i}} t_{\tilde{q}_{j}}}+\frac{\left(Q_{u u}\right)_{i j}^{22}}{u_{\tilde{q}_{i}} u_{\tilde{q}_{j}}}+\frac{\left(Q_{t u}\right)_{i j}^{22}}{t_{\tilde{q}_{i}} u_{\tilde{q}_{j}}}\right] \\
& +\left(1-h_{a}\right)\left(1-h_{b}\right)\left[\frac{\left(Q_{t t}\right)_{i j}^{12}}{t_{\tilde{q}_{i}} t_{\tilde{q}_{j}}}+\frac{\left(Q_{u u}\right)_{i j}^{12}}{u_{\tilde{q}_{i}} u_{\tilde{q}_{j}}}+\frac{\left(Q_{t u}\right)_{i j}^{12}}{t_{\tilde{q}_{i}} u_{\tilde{q}_{j}}}\right] \\
& \left.+\left(1+h_{a}\right)\left(1+h_{b}\right)\left[\frac{\left(Q_{t t}\right)_{i j}^{21}}{t_{\tilde{q}_{i}} t_{\tilde{q}_{j}}}+\frac{\left(Q_{u u}\right)_{i j}^{21}}{u_{\tilde{q}_{i}} u_{\tilde{q}_{j}}}+\frac{\left(Q_{t u}\right)_{i j}^{21}}{t_{\tilde{q}_{i}} u_{\tilde{q}_{j}}}\right]\right\},
\end{aligned}
$$

where we have introduced the generalized charges,

$$
\begin{aligned}
\left(Q_{t t}\right)_{i j}^{m n}= & \mathcal{C}_{\tilde{q}_{i} q \tilde{g}}^{m *} \mathcal{C}_{\tilde{q}_{i} q^{\prime} \tilde{\chi}_{i}}^{n *} \mathcal{C}_{\tilde{q}_{j} q \tilde{g}}^{m} \mathcal{C}_{\tilde{q}_{j} q^{\prime} \tilde{\chi}_{i}}^{n} t_{\tilde{g}} t_{\tilde{\chi}_{i}}, \\
\left(Q_{u u}\right)_{i j}^{m n}= & \mathcal{C}_{\tilde{q}_{i} q^{\prime} \tilde{g}^{\prime}}^{n *} \mathcal{C}_{\tilde{q}_{i} q \tilde{\chi}_{i}}^{m *} \mathcal{C}_{\tilde{q}_{j} q^{\prime} \tilde{g}}^{n} \mathcal{C}_{\tilde{q}_{j} q \tilde{\chi}_{i}}^{m} u_{\tilde{g}} u_{\tilde{\chi}_{i}}, \\
\left(Q_{t u}\right)_{i j}^{m n}= & 2 \operatorname{Re}\left[\mathcal{C}_{\tilde{q}_{i} q \tilde{g}}^{m} \mathcal{C}_{\tilde{q}_{i}}^{n} q^{\prime} \tilde{\chi}_{i} \mathcal{C}_{\tilde{q}_{j}}^{n} q^{\prime} \mathcal{C}_{\tilde{q}_{j}}^{m} \tilde{\chi}_{i} u_{\tilde{g}} u_{\tilde{\chi}_{i}}\right]( \\
& \left.\times\left(1-\delta_{m n}\right)\left(u t-m_{\tilde{g}}^{2} m_{\tilde{\chi}_{i}}^{2}\right)-\delta_{m n} m_{\tilde{g}} m_{\tilde{\chi}_{i}} s\right) .
\end{aligned}
$$

\section{Numerical predictions for NMFV gluino production at the $\mathrm{LHC}$}

In this section, we present numerical predictions in the context of nonminimally flavor-violating supersymmetry at the LHC for cross sections related to the production of gluino pairs as well as to the one of associated pairs of a gluino and a squark, an antisquark, a chargino or a neutralino. Squarks and gluinos are expected to be copiously produced at the LHC, due to their strong couplings to quarks and gluons. However, in the case of the benchmark scenarios presented in Table I, the high mass of the colored superpartners drastically reduces the LHC sensitivity, most of the channels being hence largely phase-space suppressed. Therefore, we focus on $p p$ collisions at the
LHC design center-of-mass energy of $\sqrt{S}=14 \mathrm{TeV}$, supposed to be reached in the second phase of the running of the LHC, after the shutdown of 2013.

Thanks to the QCD factorization theorem, total hadronic production cross sections can be computed by convolving the partonic cross sections derived in Sec. IV B, summed and averaged over final and initial spins, respectively, with the universal parton densities $f_{a / p}$ and $f_{b / p}$ of partons $a, b$ in the proton, which depend on the longitudinal momentum fractions of the two partons $x_{a, b}=\sqrt{\tau} e^{ \pm y}$ and on the unphysical factorization scale $\mu_{F}$,

$$
\begin{aligned}
\sigma= & \int_{4 m^{2} / S}^{1} \mathrm{~d} \tau \int_{-1 / 2 \ln \tau}^{1 / 2 \ln \tau} \mathrm{d} y \int_{t_{\min }}^{t_{\max }} \mathrm{d} t f_{a / p}\left(x_{a}, \mu_{F}\right) f_{b / p}\left(x_{b}, \mu_{F}\right) \\
& \times \frac{\mathrm{d} \hat{\sigma}}{\mathrm{d} t} .
\end{aligned}
$$

Neglecting all quark masses but the top mass, we employ the leading order (LO) set of the CTEQ6 parton density fit [64], which includes $n_{f}=5$ light quark flavors and the gluon, but no top-quark density. Consistently, the strong coupling constant $g_{s}$ is evaluated with the corresponding $\mathrm{LO}$ value of the QCD scale $\Lambda_{\mathrm{LO}}^{n_{f}=5}=165 \mathrm{MeV}$. For all our results, we identify the renormalization scale $\mu_{R}$ with the 
TABLE III. Cross sections, in fb, for the production of pairs of gluinos ( $p p \rightarrow \tilde{g} \tilde{g})$, for the one of associated pairs of gluinos and charginos $\left(p p \rightarrow \tilde{g} \tilde{\chi}_{i}^{+}+\tilde{g} \tilde{\chi}_{i}^{-}\right.$for $\left.i=1,2\right)$ and for the one of associated pairs of gluinos and neutralinos $\left(p p \rightarrow \tilde{g} \tilde{\chi}_{i}^{0}\right.$ for $i=1,2,3$, 4), for the reference scenarios presented in Table I.

\begin{tabular}{l|c|cc|cccc}
\hline \hline & $\tilde{g} \tilde{g}$ & $\tilde{g} \tilde{\chi}_{1}^{ \pm}$ & $\tilde{g} \tilde{\chi}_{2}^{ \pm}$ & $\tilde{g} \tilde{\chi}_{1}^{0}$ & $\tilde{g} \tilde{\chi}_{2}^{0}$ & $\tilde{g} \tilde{\chi}_{3}^{0}$ & $\tilde{g} \tilde{\chi}_{4}^{0}$ \\
\hline SPS9 & 93.8 & 43.8 & 0.055 & 5.1 & 0.46 & 0.006 \\
I & 76.5 & 29.0 & 0.040 & 3.3 & 0.001 \\
II & 58.9 & 10.8 & 0.022 & 1.2 & 0.19 & 0.004 & 0.002 \\
III & 52.5 & 4.2 & 0.014 & 0.47 & 0.085 & 0.001 \\
\hline \hline
\end{tabular}

factorization scale $\mu_{F}$ and set the scales to the average mass of the final state supersymmetric particles $m$.

For gluino pair production as well as for the associated production of a chargino or a neutralino with a gluino, only the $t$ - and $u$-channel diagrams depend on the flavorviolating parameters in the squark sector since they contain a squark propagator (see the Feynman diagrams shown in Figs. 11 and 13). However, all squark eigenstates contribute to the total cross section and the corresponding diagrams must be summed over, leading subsequently to production cross sections insensitive to nonminimal flavor violation. We present these results, therefore independent of the $\lambda$ parameters, in Table III, both for our scenarios I, II and III as well as for the SPS9 benchmark point as a reference. Strong gluino pair production is clearly dominant, and the luminosity required to observe possible signal events is not so high. In contrast, the cross sections related to the semiweak production of a gluino and a chargino/ neutralino vary from $\mathcal{O}(10)$ fb for the lightest chargino and neutralino case to the barely visible level of $\mathcal{O}\left(10^{-3}\right)$ fb for the heavier superpartners.

Contrary to the previous cases, associated squark and gluino production shows an interesting dependence on the nonminimal flavor-violation $\lambda$ parameters, as illustrated in Figs. 14-16 for our scenarios I, II and III, respectively. Moreover, the cross sections are fairly high, reaching the
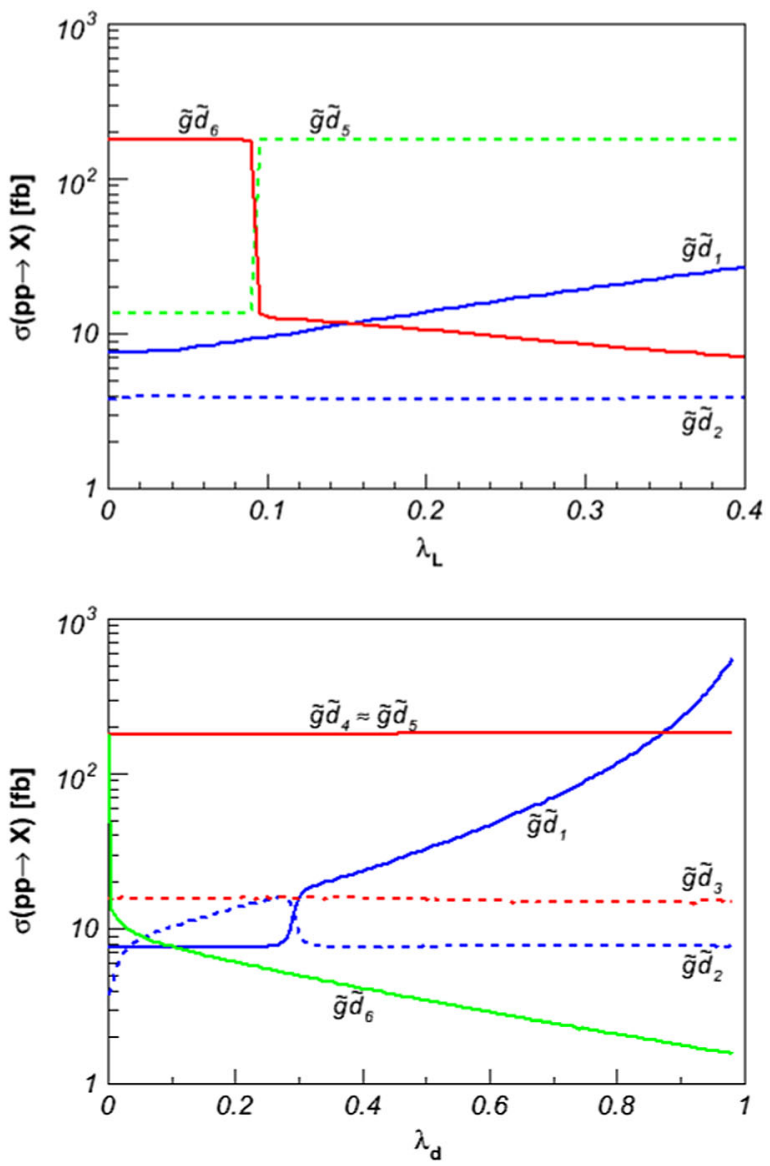

FIG. 14 (color online). Cross sections of gluino production in association with up- and down-type squarks for various ranges of the flavor-mixing parameters $\lambda_{L}, \lambda_{u}$, and $\lambda_{d}$ for the reference scenario I of Table I. 

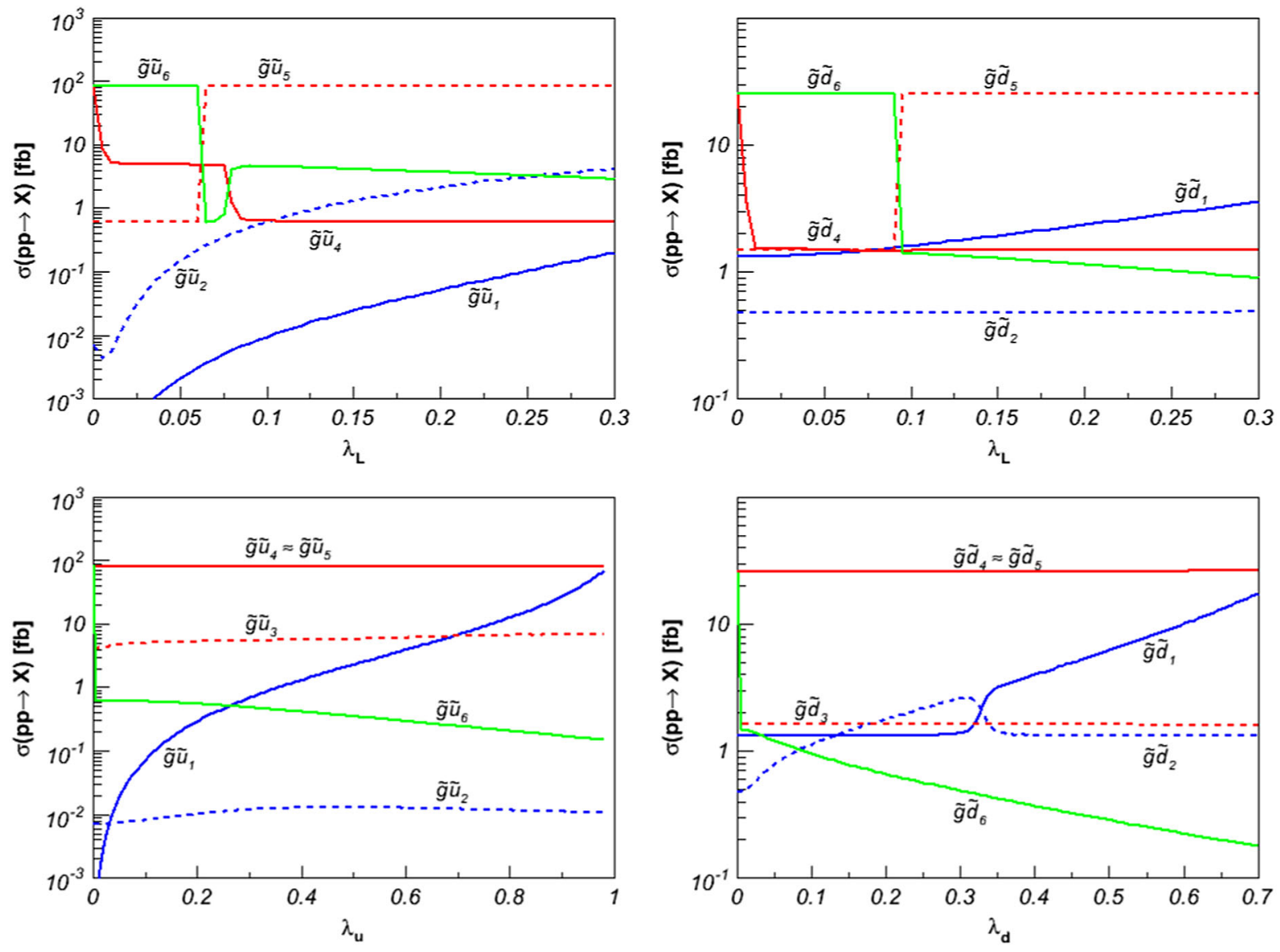

FIG. 15 (color online). Same as Fig. 14 for reference scenario II.

level of several hundereds of fb for many channels, which makes them nice candidates to study nonminimal flavor violation in supersymmetry at the LHC. Analyzing the dependence of the cross section on the $\lambda$ parameters, sharp and smooth transitions can be observed, the first ones being related to the presence of an avoided crossing and the second ones to a smooth change in the flavor content of the relevant eigenstate (see Sec. III C). Indeed, at the point where the mass of two eigenstates should cross, e.g., at $\lambda_{\mathrm{L}} \sim 0.07$ on the top-left panel of Fig. 14 or at $\lambda_{d} \sim 0.32$ on the lower-right panel of Fig. 15, the flavor content of both squarks is exchanged and the same sharp transition is observed at the level of the cross section. Smooth increases and decreases in the cross section with the values of the $\lambda$ parameters have two sources. First-, second- and thirdgeneration squark mixing induces larger mass splitting, as illustrated in Figs. 5-7. This renders certain channels phase-space favored and other channels phase-space suppressed. Secondly, the magnitude of the cross section is connected to the flavor content of the squark produced in association with the gluino, since producing a given flavor of squark requires an initial quark of the same flavor, as shown in the Feynman diagrams of Fig. 12. As an example, the lightest down-type squark mass eigenstate is a pure sbottom state for small values of $\lambda_{d} \lesssim 0.3$, while it be- comes a mixed state for $\lambda_{d} \gtrsim 0.3$, with a larger and larger strange squark component with increasing values of $\lambda_{d}$, as it can be seen from the upper-right panel of Fig. 5. Consequently, as presented in lower-right panel of Fig. 14, the cross section related to the process $p p \rightarrow$ $\tilde{g} \tilde{d}_{1}$ is small and constant for $\lambda_{d} \lesssim 0.3(\sigma \approx 8 \mathrm{fb})$ and gets larger and larger for increasing $\lambda_{d}$, even reaching a couple of hundreds of fb for $\lambda_{d} \gtrsim 0.8$. The opposite effects can be observed for the process $p p \rightarrow \tilde{g} \tilde{u}_{6}$ in the context of the scenario III, as shown in the lower-left panel of Fig. 16.

\section{COSMOLOGICAL ASPECTS}

Among the most compelling evidences for physics beyond the standard model is the presence of cold dark matter (CDM) in our Universe. Its relic density is today constrained to be

$$
\Omega_{\mathrm{CDM}} h^{2}=0.1123 \pm 0.0035
$$

from recent WMAP data combined with measurements related to supernovae and baryonic acoustic oscillations [65]. Here, $h$ is the present Hubble expansion rate in units of $100 \mathrm{~km} \mathrm{~s}^{-1} \mathrm{Mpc}^{-1}$. New physics models should therefore include a viable dark matter candidate that can account for the above amount of dark matter. 

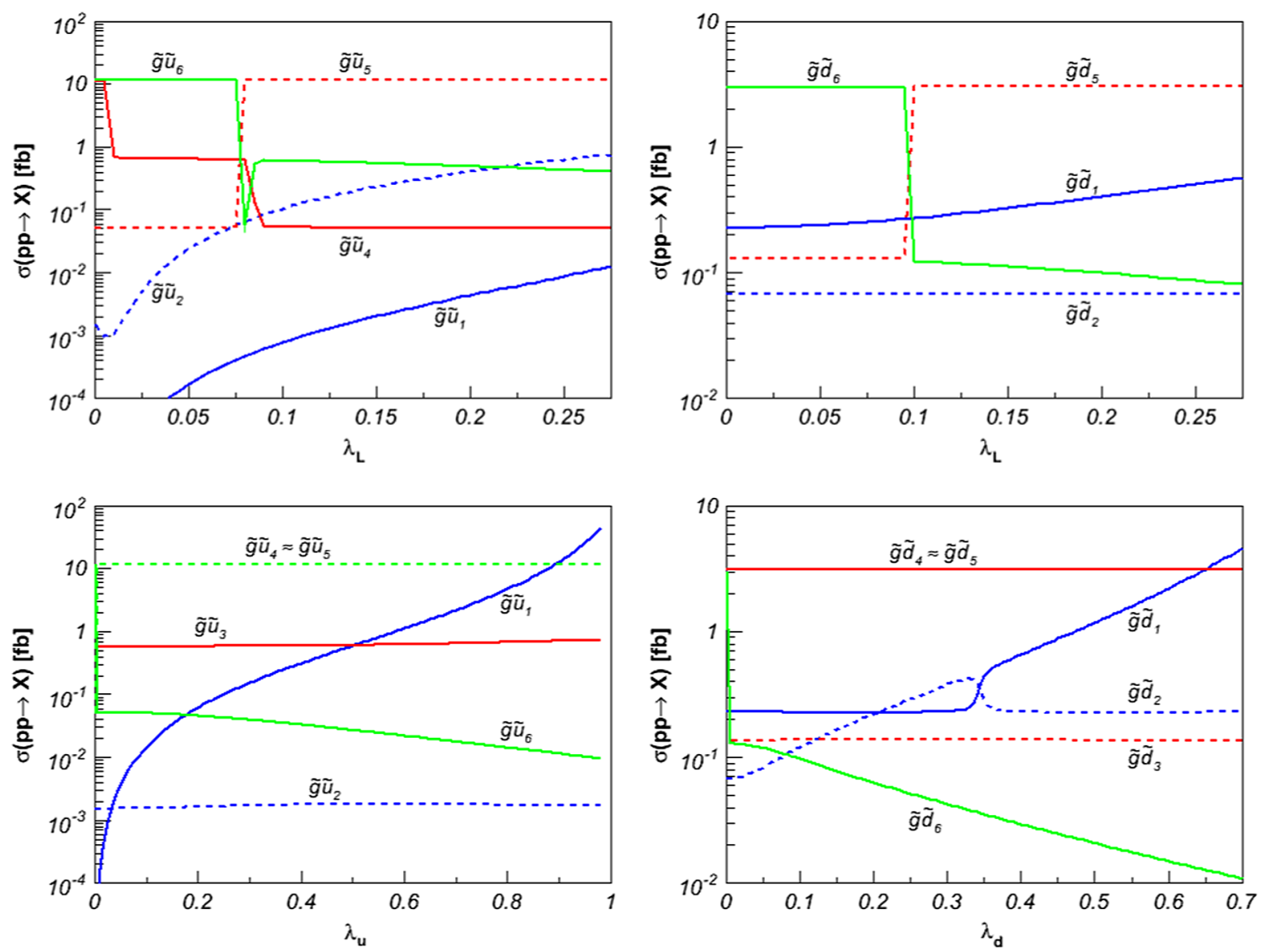

FIG. 16 (color online). Same as Fig. 14 for reference scenario III.

In AMSB models, the lightest of the four neutralinos is the lightest superpartner and therefore the dark matter candidate, if $R$ parity is assumed to be conserved. After the renormalization-group evolution from the high-scale parameters to the weak scale, the wino mass parameter $M_{2}$ turns out to be smaller than the bino and gluino masses $M_{1}$ and $M_{3}$. In consequence, in AMSB scenarios the lightest neutralino is winolike. This is in contrast to, e.g., models based on minimal supergravity where usually $M_{1}<M_{2}$ leading to a binolike LSP. Since also the chargino mass is governed by $M_{2}$, the mass difference between the lightest neutralino and the lightest chargino is rather small. For our reference scenarios, the mass difference is less than a $\mathrm{GeV}$, as can be seen in Table I. Because of the larger pair annihilation cross section as compared to the bino and due to efficient coannihilations with the chargino, the resulting relic density of the thermally produced neutralino is usually one or 2 orders of magnitude below the range given in Eq. (5.1) $[66,67]$. Using the public program DARKSUSY [68], we obtain the values $\Omega_{\tilde{\chi}_{1}^{0}} h^{2}=8.57 \times 10^{-4}, 7.87 \times$ $10^{-4}, 7.83 \times 10^{-4}$, and $7.83 \times 10^{-4}$ for the scenarios of Table I, respectively.

However, thermal production of neutralinos is not the only mechanism to be considered. Possible nonthermal production modes include the decay of heavy fields such as moduli or gravitinos in the early universe [67,69]. Moreover, axions and axinos can contribute to the dark matter relic abundance [70,71]. The contribution to the neutralino relic density from moduli decay can be estimated as [72]

$$
\begin{aligned}
\Omega_{\tilde{\chi}_{1}^{0}} h^{2} \simeq & 0.1\left(\frac{m_{\tilde{\chi}_{1}^{0}}}{100 \mathrm{GeV}}\right)\left(\frac{10.75}{g_{*}}\right)^{1 / 4}\left(\frac{3 \times 10^{-24} \mathrm{~cm}^{3} / \mathrm{s}}{\langle\sigma v\rangle}\right) \\
& \times\left(\frac{100 \mathrm{TeV}}{m_{\Phi}}\right)^{3 / 2} .
\end{aligned}
$$

It depends on the neutralino mass $m_{\tilde{\chi}_{1}^{0}}$, the mass of the moduli fields $m_{\Phi}$, the effective number of degrees of freedom $g_{*}$, and the thermally averaged annihilation cross section $\langle\sigma v\rangle$. In Fig. 17, we depict isolines of the neutralino relic density in the $m_{\tilde{\chi}_{1}^{0}}-m_{\Phi}$ plane, assuming $g_{*} \sim$ 10.75 [67] and different values of the annihilation cross section $\langle\sigma v\rangle$. Our typical scenarios lead to a neutralino relic density of about $\Omega_{\tilde{\chi}_{1}^{1}} h^{2}=8 \times 10^{-4}$, which is consistent with a neutralino annihilation cross section of $\langle\sigma v\rangle \sim 10^{-23} \mathrm{~cm}^{3} \mathrm{~s}^{-1}$. For such cross sections, a neutralino mass of $m_{\tilde{\chi}_{1}^{0}} \sim 175 \mathrm{GeV}$ and moduli masses comparable with the gravitino mass, $m_{\Phi} \sim m_{3 / 2} \sim 60 \mathrm{TeV}$, 

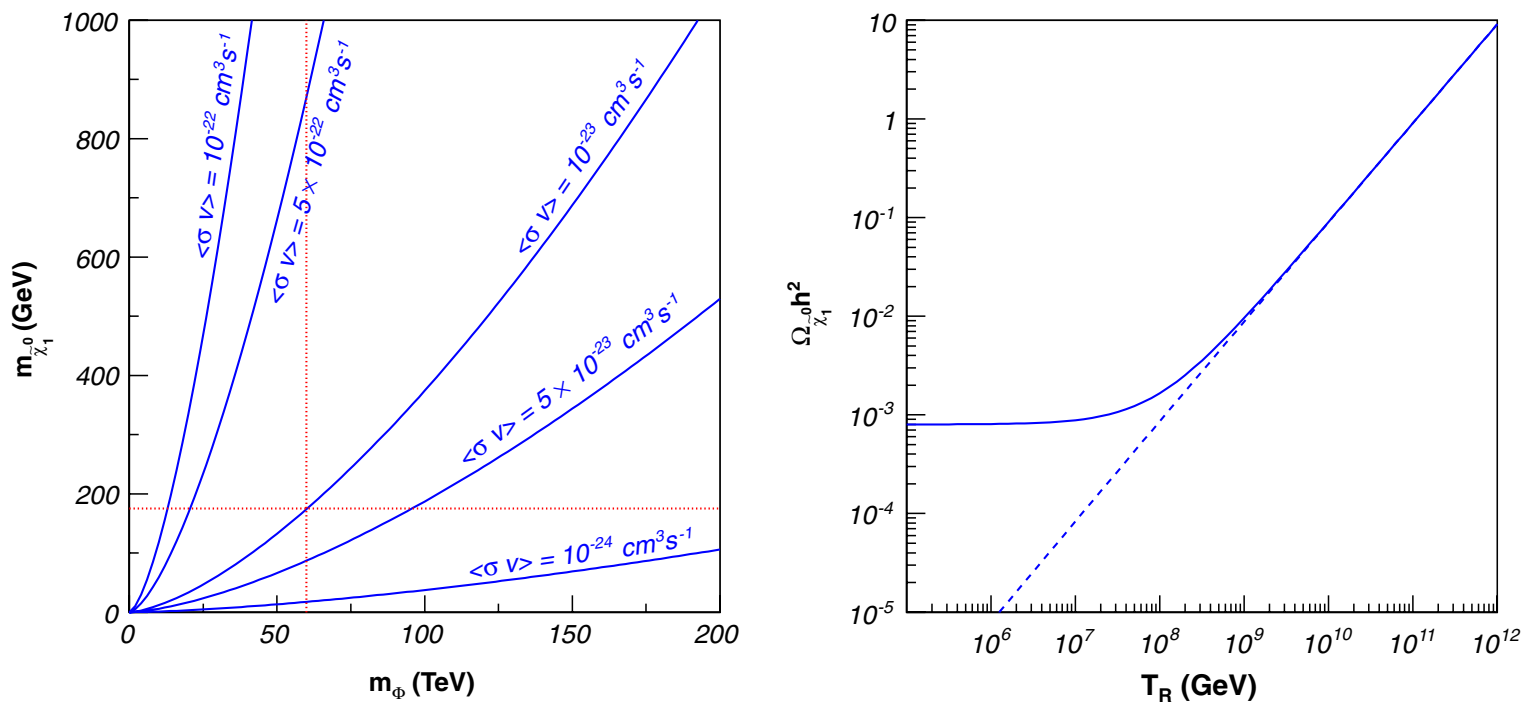

FIG. 17 (color online). Left: Isolines corresponding to $\Omega_{\tilde{\chi}_{1}^{0}} h^{2}=0.1123$ in the $m_{\Phi^{-}} m_{\tilde{\chi}_{1}^{0}}$ planes for different values of $\langle\sigma v\rangle$. The dotted lines correspond to $m_{\tilde{\chi}_{1}^{0}}=175 \mathrm{GeV}$ and $m_{\Phi}=m_{3 / 2}=60 \mathrm{TeV}$. Right: The neutralino relic density $\Omega_{\tilde{\chi}_{1}^{0}} h^{2}$ from gravitino decay as a function of the reheating temperature $T_{R}$ for a typical AMSB scenarios with $m_{\tilde{\chi}_{1}^{0}}=175 \mathrm{GeV}, m_{3 / 2}=60 \mathrm{TeV}$, as it is the case for the scenarios of Table I. The solid line includes a contribution of $\Omega_{\tilde{\chi}} h^{2}=8-10^{-4}$ from thermal neutralinos, while the dashed line indicates the contribution from gravitino decay only.

which is consistent with gravity and anomaly mediation [72], this yields nearly the measured abundance of Eq. (5.1).

The neutralino relic abundance from gravitino decay is directly related to the thermal abundance $\Omega_{3 / 2} h^{2}$, that the gravitino would have if it did not decay. The latter is obtained from computing the thermal production of gravitinos in the early universe. More precisely, the resulting neutralino relic density can then be evaluated according to [73]

$$
\begin{aligned}
\Omega_{\tilde{\chi}_{1}^{0}} h^{2}= & \frac{m_{\tilde{\chi}_{1}^{0}}}{m_{3 / 2}} \Omega_{3 / 2} h^{2} \simeq \frac{m_{\tilde{\chi}_{1}^{0}}}{m_{3 / 2}}\left(\frac{m_{3 / 2}}{100 \mathrm{GeV}}\right)\left(\frac{T_{R}}{10^{10} \mathrm{GeV}}\right) \\
& \times \sum_{i=1}^{3} \omega_{i} g_{i}^{2}\left(1+\frac{M_{i}^{2}}{3 m_{3 / 2}^{2}}\right) \log \frac{k_{i}}{g_{i}}
\end{aligned}
$$

where the ratio of neutralino and gravitino mass expresses the fact that each gravitino decays into one stable neutralino. The thermal gravitino production depends linearly on the gravitino mass $m_{3 / 2}$ and the reheating temperature $T_{R}$ of the Universe after inflation. The sum runs over the three gauge groups $U(1), S U(2)$, and $S U(3), g_{i}$ are the coupling constants of the three gauge groups, and $M_{i}$ the mass parameters of the associated gauginos. Note that in Eq. (5.3), $g_{i}$ and $M_{i}$ are evaluated at the reheating scale $T_{R}$. The constants $\omega_{i}$ and $k_{i}$ are given by $\omega_{i}=0.018$, $0.044,0.117$ and $k_{i}=1.266,1.312,1.271$ for $i=1,2,3$, respectively [73].

The right panel of Fig. 17 shows the resulting neutralino relic density as a function of the reheating temperature for the situation corresponding to the scenarios of Table I. For low values of $T_{R}$, the thermal neutralino production domi- nates, leading to the value of $\Omega_{\tilde{\chi}_{1}^{0}} h^{2}=8.57 \times 10^{-4}$ already mentioned above. For $T_{R} \geqslant 10^{7} \mathrm{GeV}$, the contribution from gravitino decay becomes dominant and $\Omega_{\tilde{\chi}_{1}^{0}} h^{2}$ grows linearly with $T_{R}$. As can be seen, the observed relic density of $\Omega_{\tilde{\chi}_{1}^{0}} h^{2} \sim 0.11$ is obtained for a reheating temperature of $T_{R} \sim 10^{10} \mathrm{GeV}$, which is well compatible with thermal leptogenesis [74].

The relic abundance of the neutralino may also depend on flavor-violating entries of the squark (or slepton) mass matrices. In Ref. [75], this has been studied for the case of minimal supergravity scenarios, where flavor-violating couplings can modify the annihilation and coannihilation modes that enter the Boltzmann equation in the typical scenario with thermal production of neutralinos. Similar conclusions have been found for flavor nondiagonal entries in the leptonic soft matrices [76]. Flavor-violating effects are, of course, also present in the discussed cases of moduli or gravitino decay. A full study of their impact within this context is, however, clearly beyond the scope of this work.

\section{CONCLUSION}

In this paper, we have studied the consequences of nonminimal flavor violation in minimal anomaly-mediated supersymmetry-breaking models, where tachyonic sleptons are avoided by introducing a common scalar mass similar to the one introduced in minimal supergravity. In these scenarios, new sources of flavor violation are in general introduced at high scales, leading to different flavor mixings for SM particles and their superpartners at the weak scale. 
Using the conventional parameterization of squark mixing at the weak scale, we analyzed the viable AMSB parameter space in the light of the latest limits on low-energy observables and of the latest results from direct searches for Higgs and SUSY particles at the LHC. We found that intermediate values of $\tan \beta=10 \ldots 30$ and relatively large scalar masses of $m_{0}=1 \ldots 3 \mathrm{TeV}$, increasing with $\tan \beta$, were preferred and allowed for sizeable flavour violation in the left-left and essentially unconstrained flavour violation in the right-right squark sectors.

We completed our analytical calculations of flavorviolating supersymmetric particle production at hadron colliders with those related to gluino pair production and to the associated production of gluinos with charginos and neutralinos as well as with squarks. The corresponding cross sections were expected to be large due to the strong coupling of gluinos to the initial quarks and gluons. Flavorviolation effects were expected to be only significant for the associated production of gluinos and squarks, since the other processes involved (almost) complete sums over internal squark exchanges.

This was confirmed in our numerical analysis for the high-energy phase of the LHC, where phenomena such as avoided crossings or smooth flavor dependences known from our previous studies could again be observed. For the experimental analysis of the ensuing cascade decays, leading to final states with second- and third-generation quarks and missing transverse energy, we referred the reader to previously published dedicated studies performed, e.g., in supergravity models. The corresponding analysis in AMSB models was beyond the scope of this paper and is left for future work.

Finally, we briefly addressed the related cosmological aspects, showing that the well-known problem of dark matter underabundance in minimal AMSB models could be solved with moduli or gravitino decays also in the presence of flavor violation.

\section{ACKNOWLEDGMENTS}

The authors would like to thank W. Porod for helpful discussions. This work has been supported by the TheoryLHC France-initiative of the CNRS/IN2P3 and by the "Helmholtz Alliance for Astroparticle Phyics HAP" funded by the Initiative and Networking Fund of the Helmholtz Association.
[1] H. P. Nilles, Phys. Rep. 110, 1 (1984).

[2] H. E. Haber and G. L. Kane, Phys. Rep. 117, 75 (1985).

[3] L. J. Hall, V. A. Kostelecky, and S. Raby, Nucl. Phys. B267, 415 (1986).

[4] G. Bozzi, B. Fuks, B. Herrmann, and M. Klasen, Nucl. Phys. B787, 1 (2007).

[5] B. Fuks, B. Herrmann, and M. Klasen, Nucl. Phys. B810, 266 (2009).

[6] A. H. Chamseddine, R. L. Arnowitt, and P. Nath, Phys. Rev. Lett. 49, 970 (1982).

[7] R. Barbieri, S. Ferrara, and C. A. Savoy, Phys. Lett. 119B, 343 (1982).

[8] M. Dine, W. Fischler, and M. Srednicki, Nucl. Phys. B189, 575 (1981); S. Dimopoulos and S. Raby, Nucl. Phys. B192, 353 (1981); C. Nappi and B. Ovrut, Phys. Lett. 113B, 175 (1982).

[9] M. Dine and A. E. Nelson, Phys. Rev. D 48, 1277 (1993); M. Dine, A. E. Nelson, and Y. Shirman, Phys. Rev. D 51, 1362 (1995); M. Dine, A.E. Nelson, Y. Nir, and Y. Shirman, Phys. Rev. D 53, 2658 (1996).

[10] G. F. Giudice and R. Rattazzi, Nucl. Phys. B511, 25 (1998); N. Arkani-Hamed, G. F. Giudice, M. A. Luty, and R. Rattazzi, Phys. Rev. D 58, 115005 (1998).

[11] G. F. Giudice and R. Rattazzi, Phys. Rep. 322, 419 (1999).

[12] K. Tobe, J. D. Wells, and T. Yanagida, Phys. Rev. D 69, 035010 (2004).

[13] S. L. Dubovsky and D. S. Gorbunov, Nucl. Phys. B557, 119 (1999).

[14] L. Randall and R. Sundrum, Nucl. Phys. B557, 79 (1999).
[15] G. F. Giudice, R. Rattazzi, M. A. Luty, and H. Murayama, J. High Energy Phys. 12 (1998) 027.

[16] T. Gherghetta, G. F. Giudice, and J. D. Wells, Nucl. Phys. B559, 27 (1999).

[17] A. Pomarol and R. Rattazzi, J. High Energy Phys. 05 (1999) 013.

[18] I. Jack and D. R. T. Jones, Phys. Lett. B 465, 148 (1999).

[19] N. Arkani-Hamed, H. Murayama, Y. Nomura, and D.E. Kaplan, J. High Energy Phys. 02 (2001) 041.

[20] J. L. Feng, T. Moroi, L. Randall, M. Strassler, and S. Su, Phys. Rev. Lett. 83, 1731 (1999).

[21] J. L. Feng and T. Moroi, Phys. Rev. D 61, 095004 (2000).

[22] R. Rattazzi, A. Strumia, and J. D. Wells, Nucl. Phys. B576, 3 (2000).

[23] A. J. Barr, C. G. Lester, M. A. Parker, B. C. Allanach, and P. Richardson, J. High Energy Phys. 03 (2003) 045.

[24] Z. Chacko, M. A. Luty, I. Maksymyk, and E. Pontón, J. High Energy Phys. 04 (2000) 001.

[25] E. Katz, Y. Shadmi, and Y. Shirman, J. High Energy Phys. 08 (1999) 015.

[26] I. Jack and D. R. T. Jones, Phys. Lett. B 482, 167 (2000).

[27] I. Jack and D. R. T. Jones, Nucl. Phys. B662, 63 (2003).

[28] B. Murakami and J. D. Wells, Phys. Rev. D 68, 035006 (2003).

[29] R. Kitano, G. D. Kribs, and H. Murayama, Phys. Rev. D 70, 035001 (2004).

[30] M. Ibe, R. Kitano, and H. Murayama, Phys. Rev. D 71, 075003 (2005).

[31] R. Hodgson, I. Jack, D. R. T. Jones et al., Nucl. Phys. B728, 192 (2005). 
[32] D. R. T. Jones and G. G. Ross, Phys. Lett. B 642, 540 (2006).

[33] R. Hodgson, I. Jack, and D. R. T. Jones, J. High Energy Phys. 10 (2007) 070.

[34] F. Gabbiani, E. Gabrielli, A. Masiero, and L. Silvestrini, Nucl. Phys. B477, 321 (1996).

[35] B. C. Allanach, G. Hiller, D. R. T. Jones, and P. Slavich, J. High Energy Phys. 04 (2009) 088.

[36] https://twiki.cern.ch/twiki/bin/view/AtlasPublic/ SupersymmetryPublicResults.

[37] https://twiki.cern.ch/twiki/bin/view/CMSPublic/ PhysicsResultsSUS.

[38] K. Nakamura et al. (Particle Data Group), J. Phys. G 37, 075021 (2010).

[39] D. Asner et al. (Heavy Flavor Averaging Group), arXiv:1010.1589.

[40] J. S. Hagelin, S. Kelley, and T. Tanaka, Nucl. Phys. B415, 293 (1994).

[41] P. Brax and C. A. Savoy, Nucl. Phys. B447, 227 (1995).

[42] M. Ciuchini, E. Franco, D. Guadagnoli, V. Lubicz, M. Pierini, V. Porretti, and L. Silvestrini, Phys. Lett. B 655, 162 (2007).

[43] T. Hurth, E. Lunghi, and W. Porod, Nucl. Phys. B704, 56 (2005).

[44] M. Misiak et al., Phys. Rev. Lett. 98, 022002 (2007).

[45] T. Huber, T. Hurth, and E. Lunghi, Nucl. Phys. B802, 40 (2008).

[46] CMS and LHCb Collaborations, CMS-PAS-BPH-11-019.

[47] P. Ball and R. Fleischer, Eur. Phys. J. C 48, 413 (2006).

[48] T. Moroi, Phys. Rev. D 53, 6565 (1996); 56, 4424(E) (1997).

[49] W. Porod, Comput. Phys. Commun. 153, 275 (2003).

[50] ATLAS Collaboration, ATLAS-CONF-2011-163.

[51] CMS Collaboration, CMS-PAS-HIG-11-032.

[52] M. Lancaster (Tevatron Electroweak Working Group and for CDF and D0 Collaboration), arXiv:1107.5255.

[53] B. C. Allanach, T. J. Khoo, and K. Sakurai, J. High Energy Phys. 11 (2011) 132.

[54] S.P. Martin and J.D. Wells, Phys. Rev. D 64, 035003 (2001).

[55] B. C. Allanach et al., Eur. Phys. J. C 25, 113 (2002).

[56] T. Hurth and W. Porod, J. High Energy Phys. 08 (2009) 087.
[57] A. Bartl, K. Hidaka, K. Hohenwarter-Sodek, T. Kernreiter, W. Majerotto, and W. Porod, Phys. Lett. B 679, 260 (2009).

[58] A. Bartl, H. Eberl, B. Herrmann, K. Hidaka, W. Majerotto, and W. Porod, Phys. Lett. B 698, 380 (2011); 700, 390(E) (2011).

[59] M. Bruhnke, B. Herrmann, and W. Porod, J. High Energy Phys. 09 (2010) 006.

[60] A. Bartl, H. Eberl, E. Ginina, B. Herrmann, K. Hidaka, W. Majerotto, and W. Porod, Phys. Rev. D 84, 115026 (2011).

[61] C. Gross and G. Hiller, Phys. Rev. D 83, 095015 (2011).

[62] W. Beenakker, R. Hopker, M. Spira, and P. M. Zerwas, Nucl. Phys. B492, 51 (1997).

[63] T. Gehrmann, D. Maitre, and D. Wyler, Nucl. Phys. B703, 147 (2004).

[64] J. Pumplin, D. R. Stump, J. Huston, H. L. Lai, P. Nadolsky, and W. K. Tung, J. High Energy Phys. 07 (2002) 012.

[65] E. Komatsu et al. (WMAP Collaboration), Astrophys. J. Suppl. Ser. 192, 18 (2011).

[66] C. H. Chen, M. Drees, and J. F. Gunion, Phys. Rev. D 55, 330 (1997); 60, 039901(E) (1999).

[67] T. Moroi and L. Randall, Nucl. Phys. B570, 455 (2000).

[68] P. Gondolo, J. Edsjö, P. Ullio, L. Bergstrom, M. Schelke, and E. A. Baltz, J. Cosmol. Astropart. Phys. 07 (2004) 008; P. Gondolo, J. Edsjö, P. Ullio, L. Bergstrom, M. Schelke, E. A. Baltz, T. Bringmann, and G. Duda, http:// www.darksusy.org.

[69] S. Rajagopalan, arXiv:1010.3384.

[70] L. Covi, J. E. Kim, and L. Roszkowski, Phys. Rev. Lett. 82, 4180 (1999).

[71] L. Covi, H. B. Kim, J. E. Kim, and L. Roszkowski, J. High Energy Phys. 05 (2001) 033.

[72] B. S. Acharya, G. Kane, S. Watson, and P. Kumar, Phys. Rev. D 80, 083529 (2009).

[73] J. Pradler and F.D. Steffen, Phys. Rev. D 75, 023509 (2007).

[74] W. Buchmuller, P. Di Bari, and M. Plumacher, Ann. Phys. (N.Y.) 315, 305 (2005).

[75] B. Herrmann, M. Klasen, and Q. Le Boulc'h, Phys. Rev. D 84, 095007 (2011).

[76] D. Choudhury, R. Garani, and S. K. Vempati, J. High Energy Phys. 06 (2012) 014. 